\title{
Mechanisms of secretion and spreading of pathological tau protein
}

\author{
Cecilia A. Brunello ${ }^{1} \cdot$ Maria Merezhko $^{1} \cdot$ Riikka-Liisa Uronen $^{1} \cdot$ Henri J. Huttunen ${ }^{1}[$
}

Received: 14 June 2019 / Revised: 10 October 2019 / Accepted: 16 October 2019 / Published online: 30 October 2019

(c) The Author(s) 2019

\begin{abstract}
Accumulation of misfolded and aggregated forms of tau protein in the brain is a neuropathological hallmark of tauopathies, such as Alzheimer's disease and frontotemporal lobar degeneration. Tau aggregates have the ability to transfer from one cell to another and to induce templated misfolding and aggregation of healthy tau molecules in previously healthy cells, thereby propagating tau pathology across different brain areas in a prion-like manner. The molecular mechanisms involved in cell-tocell transfer of tau aggregates are diverse, not mutually exclusive and only partially understood. Intracellular accumulation of misfolded tau induces several mechanisms that aim to reduce the cellular burden of aggregated proteins and also promote secretion of tau aggregates. However, tau may also be released from cells physiologically unrelated to protein aggregation. Tau secretion involves multiple vesicular and non-vesicle-mediated pathways, including secretion directly through the plasma membrane. Consequently, extracellular tau can be found in various forms, both as a free protein and in vesicles, such as exosomes and ectosomes. Once in the extracellular space, tau aggregates can be internalized by neighboring cells, both neurons and glial cells, via endocytic, pinocytic and phagocytic mechanisms. Importantly, accumulating evidence suggests that prion-like propagation of misfolding protein pathology could provide a general mechanism for disease progression in tauopathies and other related neurodegenerative diseases. Here, we review the recent literature on cellular mechanisms involved in cell-to-cell transfer of tau, with a particular focus in tau secretion.
\end{abstract}

Keywords Amyloid $\cdot$ Tau $\cdot$ Aggregation $\cdot$ Propagation $\cdot$ Prion $\cdot$ Unconventional protein secretion $\cdot$ Extracellular vesicles

$\begin{array}{ll}\text { Abbreviations } \\ \text { AD } & \text { Alzheimer's disease } \\ \text { AGD } & \text { Argyrophylic grain disease } \\ \text { CBD } & \text { Corticobasal degeneration } \\ \text { CSF } & \text { Cerebrospinal fluid } \\ \text { EM } & \text { Electron microscopy } \\ \text { ER } & \text { Endoplasmic reticulum } \\ \text { FGF2 } & \text { Fibroblast growth factor } 2 \\ \text { FTD } & \text { Frontotemporal dementia } \\ \text { FTDP-17 } & \text { Frontotemporal dementia with Parkinsonism } \\ & \text { in chromosome 17 } \\ \text { GAG } & \text { Glycosaminoglycan } \\ \text { HSPG } & \text { Heparin sulfate proteoglycans } \\ \text { IDP } & \text { Intrinsically disordered protein } \\ \text { MAPS } & \text { Misfolding-associated protein secretion } \\ \text { MT } & \text { Microtubules } \\ \text { MTBD } & \text { Microtubule-binding repeat domain } \\ & \end{array}$

Henri J. Huttunen

henri.huttunen@helsinki.fi

1 Neuroscience Center, HiLIFE, University of Helsinki, P.O. Box 63, Haartmaninkatu 8, 00014 Helsinki, Finland

$\begin{array}{ll}\text { MVB } & \text { Multivesicular body } \\ \text { NFT } & \text { Neurofibrillary tangles } \\ \text { PD } & \text { Parkinson's disease } \\ \text { PiD } & \text { Pick's disease } \\ \text { PI(4,5)P2 } & \text { Phosphatidyl inositol 4,5 phosphate } \\ \text { PFF } & \text { Pre-formed fibrils } \\ \text { PM } & \text { Plasma membrane } \\ \text { PrP } & \text { Prion protein } \\ \text { PS } & \text { Phosphatidyl serine } \\ \text { PSP } & \text { Progressive supranuclear palsy } \\ \text { TBI } & \text { Traumatic brain injury } \\ \text { UPS } & \text { Unconventional protein secretion }\end{array}$

\section{Introduction}

Demographic aging is increasing the prevalence and societal cost of dementia. The number of people living with dementia is expected to reach 130 million people by 2050 (World Alzheimer Report 2015). Most chronic neurodegenerative diseases are characterized by progressive accumulation of protein aggregates in the nervous system. A common 
neuropathological hallmark in Alzheimer's disease (AD), the most common type of aging-related dementia, is cerebral accumulation of neurofibrillary tangles (NFT), composed of aggregated tau protein, which appear to spread from one affected brain region to other areas of the brain as the disease progresses. Similar accumulation of tau inclusions in brain is observed in, e.g., frontotemporal dementia (FTD), progressive supranuclear palsy (PSP), corticobasal degeneration (CBD) and Pick's disease (PiD). Slow spreading of pathologically misfolded tau protein may also be a central event in development and progression of chronic traumatic encephalopathy (CTE), which is often associated with repeated head injuries. The cellular mechanisms of spreading of pathological proteins, such as tau, $\alpha$-synuclein, $\beta$-amyloid peptide (A $\beta$ ) and TDP-43, have been receiving increasing attention, and better understanding of the spreading mechanisms is expected to accelerate development of disease-modifying therapies for these devastating neurodegenerative diseases.

\section{Tau is a microtubule-binding protein enriched in neurons}

Tau is one of the many microtubule-associated proteins (MAP) that have an important function of regulating microtubules (MT) to ensure proper cytoskeletal organization and trafficking [1], which is particularly important in highly polarized neuronal cells whose functionality and viability depend on transport of cellular cargo to and from axonal and dendritic peripheries [2-4]. Tau has important physiological functions in regulating MT, including MT polymerization, stabilization and suppression of MT dynamics. Dysregulation of the tau-MT complex leads to tau detachment and instability and disassembly of MTs, eventually leading to perturbation of MT-dependent transport and impaired maintenance of cellular polarity and viability $[4,5]$.

Tau is present in neurons and, to a lesser degree, in glial cells $[6,7]$. Tau is mainly an axonal protein in mature neurons, but it can also be found in the nucleus, mitochondria, dendrites, synapses, and at the plasma membrane (PM) [8-13]. This localization pattern suggests that besides the main function of MT regulation, tau may have other roles in cells.

Synaptic tau has been reported to be involved in synaptic development of newborn hippocampal neurons [14]. Also, a role for tau in neuronal activity has been proposed, as tau knockout mice display impaired long-term depression in the hippocampus [11]. Tau also interacts with nucleic acids and can localize to the nucleus, and upon binding to DNA it promotes DNA stability [15], suggesting that tau may have a role in DNA protection [16]. Finally, several domains of the tau protein have the ability to interact with lipids and membranes [17], and one of tau kinases, Fyn, is mainly located in PM microdomains called lipid rafts, where tau can also be recruited [18]. It is also possible that tau localized at the PM occupies a role as a signaling regulator, via its interaction with membrane receptors $[19,20]$.

Tau is encoded by the MAPT gene, which in humans is located on the chromosome 17. Tau is composed of 16 exons which give rise to six different splicing isoforms, which span from 352 to 441 amino acids (aa) [21], depending on alternative splicing of exons 2, 3 and 10 (Fig. 1a). Exons 2 and 3 encode for 29 amino acid repeats, both located at the $\mathrm{N}$-terminal part of the protein, and alternative splicing of exons 2 and 3 or exon 2 alone produces three $\mathrm{N}$-terminal protein variants $0 \mathrm{~N}, 1 \mathrm{~N}$ (additional 29 aa) or the less abundant $2 \mathrm{~N}$ (additional 58 aa). Exon 10 encodes for one of the four possible microtubule-binding repeat domains (MTBD), which are 31-32 aa long imperfect repeat sequences, located in the C-terminal half of the tau protein and affecting both microtubule-binding affinity and fibrillization properties of tau. Splicing isoforms of tau contain either three (3R) or four (4R) MTBDs, which affects their microtubule-binding affinity $(4 \mathrm{R}>3 \mathrm{R})$ and also their propensity for aggregation. The ratio between $3 R$ and $4 R$ isoforms is developmentally regulated as human fetal tau consists mostly the shortest form 0N3R, while in the adult brain all six isoforms coexist. Also, species-specific differences exist, as for example adult mice display exclusively 4R-tau isoforms [22].

Different functional domains characterize the tau protein (Fig. 1a). The N-terminal projection domain consists of residues 1-150 (of the longest isoform) and it regulates microtubule binding even though it is not directly involved in the physical interaction [23]. Absence of the $\mathrm{N}$-terminus alters cellular localization of tau, promoting cytosol to nucleus relocalization [24]. It has also been suggested that the $\mathrm{N}$-terminal projection domain mediates PM localization of tau via annexin-2 interaction [25]. The proline-rich domain occupies the central portion of tau from residue 151-243. It represents the most disordered part of the protein and serves as an interaction site for Src homology-3 (SH3) proteins, in particular the Fyn kinase [26], as well as an interaction site for DNA and RNA [27, 28]. The MTBDs, as the name suggests, interact with microtubules but also with actin, orchestrating the order and stability of the cytoskeleton. Several proteins associated with neurodegenerative disorders, including $\alpha$-synuclein, presenilin-1, FUS, and TIA1 , interact with the MTBDs and the proline-rich domains of tau, suggesting that the structural composition and protein-protein interactions of tau could play an important role in pathological processes [29].

Mutations in the MAPT gene have been linked to hereditary dominant frontotemporal dementia with parkinsonism in chromosome 17 (FTDP-17), which includes diverse clinical syndromes as well as diverse anatomical distribution of tau inclusions depending on the specific mutations [30]. First mutations in the MAPT gene were found in 1998 [31, 32] 
Fig. 1 The structural basis of tau function and aggregation. a The domain structure of human tau protein. Location of the projection domain, proline-rich domain, MTBDs, and the parts of tau protein encoded by the alternatively spliced exons 2 , 3 and 10 are shown on top of the longest tau isoform $(2 \mathrm{~N} 4 \mathrm{R}$, $441 \mathrm{aa})$. Below the location of key phosphorylated residues, the two hexapeptides, the two cysteines and examples of FTLD-associated mutations in the MTBDs are shown. b Microtubules (blue and green) are formed by the assembly of $\alpha$ - and $\beta$-tubulin dimers into protofilaments that associate laterally into hollow tubes. Tau (red) binds to the surface of microtubules interacting with $\alpha$ and $\beta$-tubulin via the MTBDs. The image was prepared based on a cryo-EM structure of microtubule-associated synthetic tau (PDB: 6CVN). c Structure of a paired helical filament (PHF) fragment isolated from AD brain. The filaments are formed of anti-parallel $\beta$-sheets, with the protofilament core formed by the four MTBDs of tau. The location of the tau hexapeptide sequence is indicated. Image was prepared based on a cryo-EM structure (PDB: 5O3L)
A Projection domain Pro-rich region Microtubule-binding repeat domains

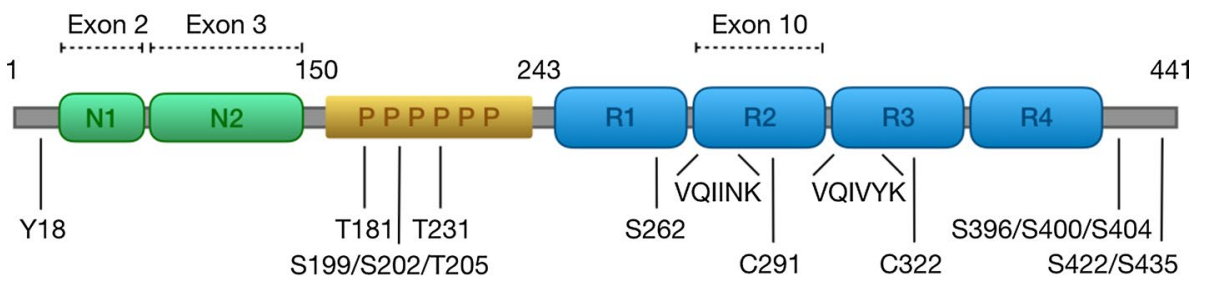

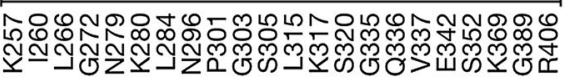

Pathogenic mutations in FTLD

B

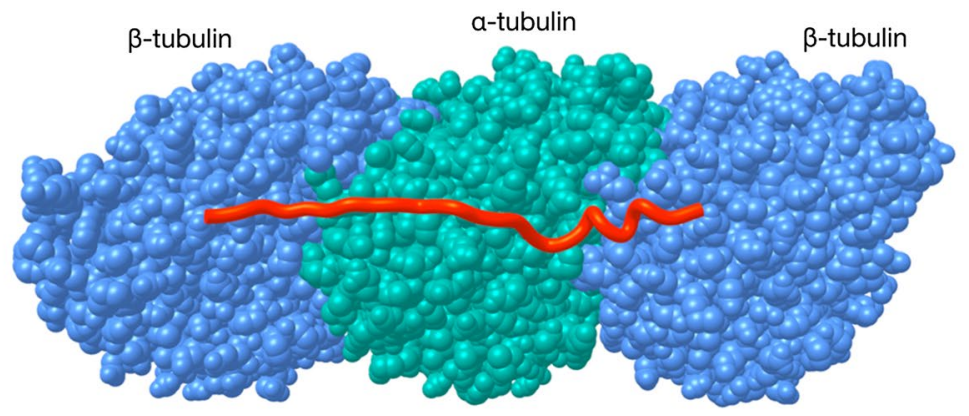

C KVQIINKKLDLSNVQSKCGSKDNIKHV
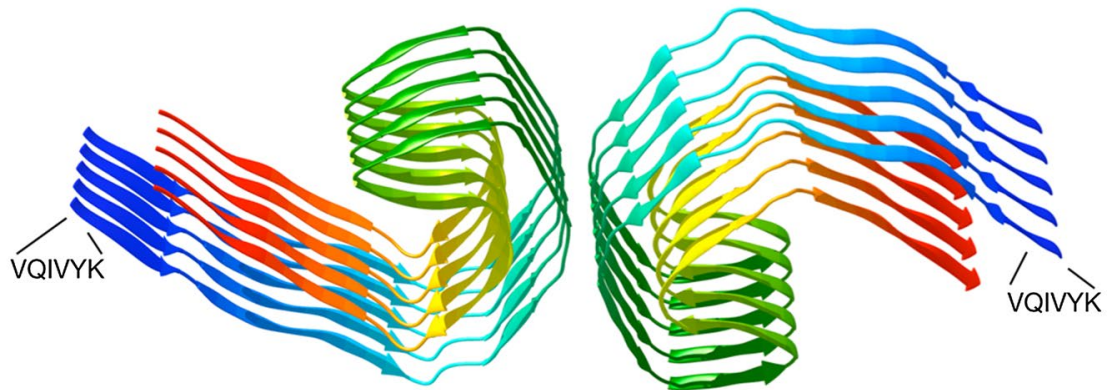

VQIVYKPVDLSKVTSKCGSLGNIHHKPGGGQVEVKSEKLDFKDRVQSKIGSLDNITHVPGGGNKKIETHKLTF linked to dominant hereditary FTDP-17, and today there are over 50 known pathogenic mutations in the MAPT gene, mostly located in exons 9-12 [30]. Such mutations can affect tau both at the protein level, for example by altering microtubule-binding properties (e.g., K257T, G272V, P301L, V337M and R406W mutations), and at the RNA level, shifting the splicing toward overproduction of 4R-tau (e.g., N279K, N296H, P301S, S305I mutations) [33, 34]. This is relevant because isoforms containing exon 2 and exon 10 are more aggregation prone compared to isoforms containing only exon 3, due to the presence of the VQIINK hexapeptide (encoded by exon 10) that mediates homo-oligomerization $[35,36]$. Additionally, alterations in the ratio of physiologically produced 3R-tau and 4R-tau may cause tau dysfunction in cells, even in the absence of mutations affecting splicing of exon 10. For instance, 4R-tau binds to microtubules with higher affinity compared to the $3 \mathrm{R}$ isoforms, promoting microtubule assembly and oversaturation of the available binding sites [37]. In turn, an imbalance between 4R-tau and 3R-tau isoforms may cause accumulation of unbound 4R-tau in the cytosol, which would favor tau aggregation.

\section{Structural plasticity and aggregation of tau}

Although tau is considered a natively unfolded protein [sometimes also called an intrinsically disordered protein (IDP)], its ability to adopt various structural states is important to its functions and pathological properties. Since the first report on tau structure in 1977 [38], there have been numerous approaches and reports addressing tau structure in various contexts (reviewed in [39]). While the intrinsically disordered nature of tau precludes the use of crystallographic methods, circular dichroism, nuclear magnetic resonance (NMR) spectroscopy, and more recently cryogenic 
electron microscopy (cryo-EM) have provided insight into conformational states of tau, in soluble physiological state, bound to microtubules or in the form of pathological aggregated states. It was also recently suggested that under physiological conditions tau monomers may adopt stable yet diverse conformations, opening a new perspective for understanding the plasticity of tau structure in physiology and in pathology [40, 41].

Multiple tau monomers bind in tandem to the surface of microtubule (MT) protofilaments. The conserved MTBDs adopt similar extended structures along the microtubules, stabilizing the interface between tubulin dimers [42] (Fig. 1b), while the $\mathrm{N}$-terminal projection domain extends away from the MT protofilament surface. While microtubule binding does not promote adoption of distinct secondary structures, the MTBDs may have some $\alpha$-helical propensity. Also, interaction with membranes may facilitate adoption of temporary secondary structures.

The ability of tau to aggregate is encoded in its own sequence. Especially, two hexapeptides present in the MTBDs (VQIVYK and VQIINK) are fundamental for aggregation and formation of the tau filaments [36, 43]. One of the hexapeptides (VQIVYK) is present in all tau isoforms in the third MTBD, while the other one (VQIINK), since it is located in the second MTBD encoded by exon 10, is only present in the $4 \mathrm{R}$ isoforms. This partially explains the higher propensity of $4 \mathrm{R}$ isoforms to aggregate compared to $3 \mathrm{R}$ isoforms. Local structural changes of the MTBDs via exposure of the VQIVYK/VQIINK motifs may determine the conversion of an inert tau monomer into a pathologically aggregated form [40]. Tau dimerization, the first step in tau oligomerization, can occur through any combination of these two motifs, which form the nucleation center where further tau dimers and tau monomers can be recruited to eventually constitute oligomers. Of particular importance are also two cysteine residues located in R2 and R3, which form disulfide bridges during tau dimerization and which have been found to constitute a critical step in the formation of tau fibrils [44]. Elongated oligomers then acquire a more ordered $\beta$-sheet structure, and finally form paired helical filaments (PHFs), which represent the building blocks of the neurofibrillary tangles (NFT) [45]. Cryo-EM structures of tau filaments extracted from AD brain confirmed the $\beta$-sheet-dominant pathological conformation [46], confirming previous findings [47]. Straight and paired helical filaments appear to be ultrasctructural polymorphs, both sharing the same basic structural architecture (Fig. 1c).

The widely studied P301L mutation, found in some patients with FTDP-17, occurs near one of the hexapeptides and promotes tau aggregation [31]. On the other hand, artificial mutations in the hexapeptides, such as proline insertion that distort the $\beta$-sheet structure, reduce tau aggregation [48]. In addition, the cellular environment, such as the presence of anionic membranes, also promotes tau aggregation $[49,50]$. A similar effect results also from polyanions such as heparin and RNA [51, 52].

Among the many post-translational modifications that modulate tau functions, phosphorylation is of particular interest because of its role in the pathological processes, as the deposits of insoluble tau found in the brains of tauopathy patients contain hyperphosphorylated tau [53-56]. Since the first report that classified tau as a phospho-protein [38], there are now at least 85 known phosphorylation sites (mostly serines and threonines, but also tyrosines). Tau is physiologically constantly phosphorylated and dephosphorylated to ensure regulation of proper functions, but when the balance is shifted toward phosphorylation, tau affinity for microtubules decreases [57]. This results in an increase in cytosolic tau, which becomes more vulnerable to aggregation [58]. Additionally, abnormally phosphorylated tau is able to sequester other microtubule-associated proteins (MAP), further worsening microtubule destabilization [57]. It is now well established that there is a strong connection between aberrant phosphorylation and self-aggregation of tau into oligomers and higher-order aggregates [36], despite the fact that a complete pathological phosphorylation pattern has not been identified yet. The neurodegeneration-associated consequences of tau hyperphosphorylation also include impaired axonal transport [59], cellular relocalization of tau to the somatodendritic compartment and synaptic loss [60]. Synaptic dysfunction can occur both pre-synaptically, where phosphorylated tau has been shown to interfere with synaptic vesicles [61], and post-synaptically via downregulation of AMPA receptors [62]. Interestingly, it was shown that synaptic terminals of AD brains and transgenic P301L mice do not contain more tau compared to control brains, indicating that there is no relocalization to synapses in pathological conditions. Instead, synaptic tau was hyperphosphorylated, suggesting a role for phosphorylation in the spreading of the disease as well [63, 64]. Additionally, hyperphosphorylation is linked to impaired degradation of tau by the ubiquitin-proteasome system [65] and to tau secretion. It was in fact shown that abnormally phosphorylated tau is secreted more efficiently than non-phosphorylated tau, at least in cell lines $[66,67]$. Finally, abnormal tau phosphorylation impairs its ability to interact with tau partners, therefore altering normal physiological properties of tau [68].

Tau phosphorylation state is a result of a tightly regulated balance of actions between many cellular proteins: mainly kinases that phosphorylate tau and phosphatases that dephosphorylate it. Tau kinases are divided into three different groups: (1) serine/threonine proline-directed kinases, which include GSK3, CdK5, MAPKs and other stressactivated kinases; (2) serine/threonine non-proline-directed kinases, which include DYRK1A, PKA, CaMKII and CK1; and (3) tyrosine kinases, such as Fyn and Src [69]. Some 
kinases, such as GSK3 $\beta$, MAPK, CK1 $\delta$ and Cdk5, are found in neurofibrillary tangles, suggesting a direct link between phosphorylation and disease progression [55]. The activity of phosphatases like PP2A, one of the principal neuronal protein phosphatases, is dramatically decreased in the brain of $\mathrm{AD}$ patients [70]. This suggests that not only upregulated kinase activity, but also downregulated phosphatase activity is involved in pathological phosphorylation of tau. There likely exist complex regulatory loops that maintain tau phosphorylation in homeostasis. For instance, impairment of the Akt/mTOR pathway may alter the physiologic phosphorylation balance between GSK3 $\beta$ and PP2A, as Akt inhibits GSK3 $\beta$, which in turn inhibits PP2A [22].

Our understanding of the intricate mechanism of tau phosphorylation equilibrium is further complicated by an increasing amount of evidence that challenge the classical view that correlates hyperphosphorylation with neurodegeneration [71]. For instance, phosphorylation sites that promote tau disassembly from the microtubules not only favor tau aggregation, but can actually inhibit it [72]. In line with these results, Arendt and colleagues suggested that hyperphosphorylation of tau may have a physiological role, possibly related to synaptic plasticity [73].

These observations lead to a very important conclusion, which was suspected for a long time but only recently experimentally proven: considering all the factors that affect tau aggregation (mutations, isoform composition, post-translational modifications and localization), it seems unlikely that a single form of tau is responsible for its pathological effects. It is more likely that a plethora of different pathological tau species with different properties accounts for the heterogeneity observed in tau-mediated pathology [74]. Moreover, tau pathology is rarely exclusively present in any of the tauopathies, as other pathological proteins and other phenotypes often accompany tau pathology and dysfunction. There may therefore be some common pathways that bring together different proteins with very different cellular functions, possibly causally linking development of distinct pathological features and resulting in synergistic modes of toxicity. For example, in mouse model of $\mathrm{AD}$, soluble $\mathrm{A} \beta$ promotes development of tau pathology [75, 76]. In tau, presenilin1 and APP transgenic mice, amyloid pathology precedes development of NFT pathology [77]. Tau and $\alpha$-synuclein promote fibrillization of each other [78] and insoluble forms of tau and $\alpha$-synuclein have been found co-aggregating in Lewy bodies, the characteristic pathological deposits of Parkinson's disease (PD) [79]. Moreover, genetic studies have linked variants of the tau gene, MAPT, to susceptibility of developing sporadic PD [80, 81], and mutations in MAPT are associated with dementia with parkinsonism [31]. It is therefore clear that the neuropathological features that characterize different tauopathies could originate from different types of tau modifications and interactions with other cellular proteins, defining different strains of pathological tau with different molecular properties.

\section{Tau inclusions in neurodegenerative diseases}

Tau dysfunction can lead to a wide variety of human disorders, called tauopathies, which include very different clinical syndromes and a constellation of pathological hallmarks. The principal tauopathies include AD, FTDP-17, the spectrum corticobasal degeneration/progressive supranuclear palsy (CBD/PSP), Pick's disease and argyrophilic grain disease (AGD). A list of the main tauopathies, with their key features, is presented in Table 1.

Tauopathies are typically associated with dementia and motor impairments, but the clinical picture can vary between different disorders and even within syndromes, ranging from minor cognitive impairments [82] to the severe behavioral changes and cognitive and motor impairments of FTDP-17 [83]. Similarly, while it is quite common for the majority of tauopathies to be characterized by generalized cortical atrophy and ventricle enlargement, which partially explains the overlapping symptoms, the progression pattern and affected brain areas may vary [84]. While biochemical, genetic, pathological and clinical similarities between tauopathies can facilitate understanding of the general principles of how tau pathologies develop, they also complicate the diagnosis and classification of different tauopathies.

Tauopathies can be classified as primary or secondary, depending whether tau inclusions constitute the main pathological hallmark or whether other pathological proteins significantly contribute to the pathogenesis of the disease [84]. For instance, $\mathrm{AD}$, the most common neurodegenerative disease and one of the main models for the investigation of tau pathology, is a secondary tauopathy to amyloid plaques, which are largely composed of aggregated $\mathrm{A} \beta$ peptides [85]. Primary tauopathies include FTDP-17, CBD/PSP, PiD and AGD. Majority of tauopathies are sporadic, but for example FTDP-17 is strictly inherited and all the different clinical and neuropathological subtypes of FTDP-17 are caused by different mutations in the MAPT gene [86]. Notably, regarding the classification of nervous system diseases, it was recently proposed that the term FTDP-17 should be retired and FTDP-17 cases should be considered as familial forms of frontotemporal lobar degeneration with tau-immunoreactive inclusions (FTLD-tau) [87].

Classification of tauopathies can also be done based on the tau isoforms present in the inclusions and morphology of the tau filaments found in the pathological deposits. While AD and FTDP-17 are mixed 3R and 4R tauopathies, reflecting the physiological ratio of expressed tau isoforms in the healthy brain [88], CBD/PSP, and AGD are classified as $4 \mathrm{R}$ tauopathies, as the pathological inclusions are predominantly constituted by $4 \mathrm{R}$-tau [84]. The only 


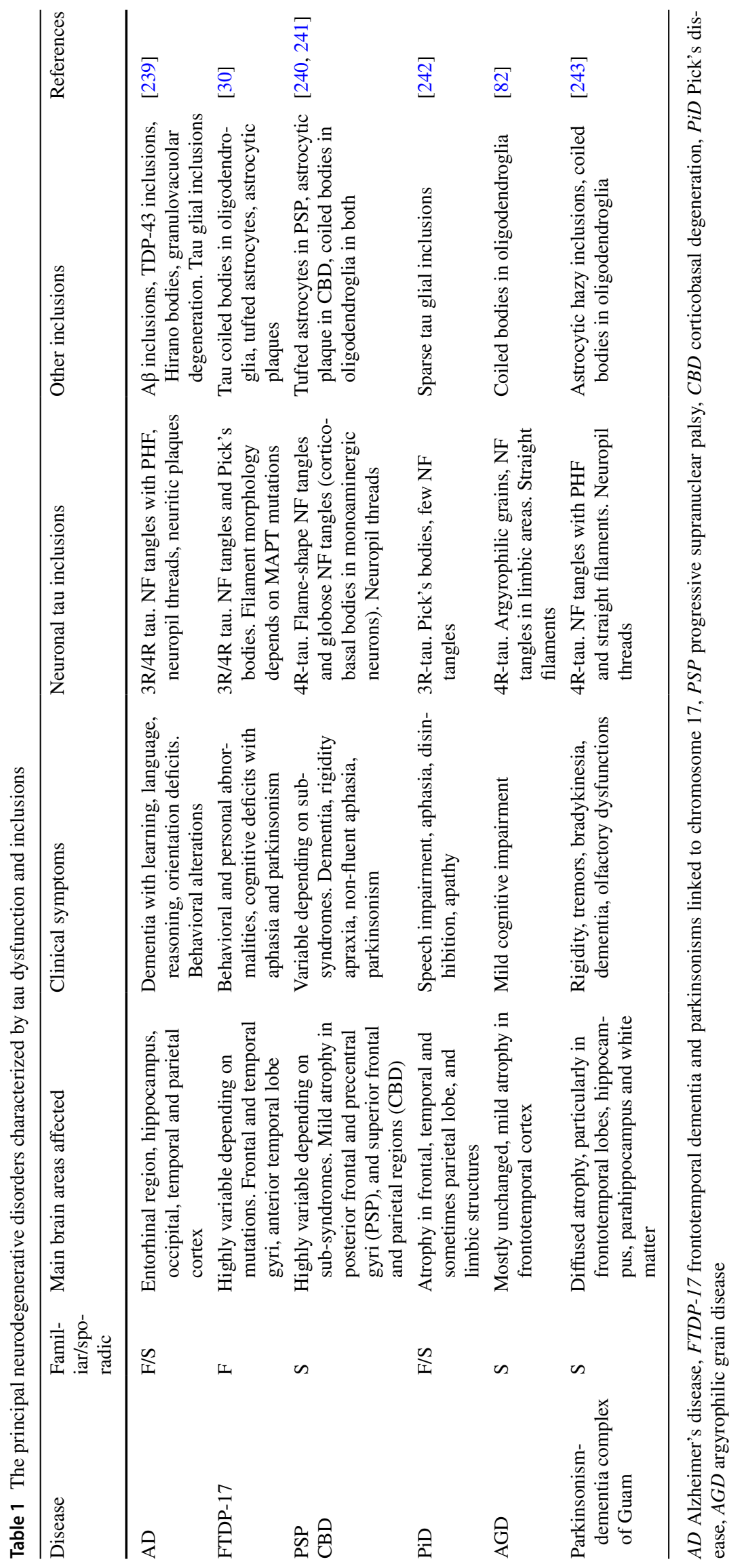


known tauopathy characterized by inclusions of $3 \mathrm{R}$-tau is Pick's disease [89]. Tau inclusions also differ regarding the morphology of tau filaments and the cell types where they are deposited. The evidence that different missense mutations in the MAPT gene can lead to different filament morphology gives an idea of the possible morphological heterogeneity of tau aggregates. For instance, N279K mutation promotes formation of tau bundles of varying thickness [90], while P301L mutation causes accumulation of twisted ribbons and stranded filaments [32] and S305I mutation promotes formation of straight and tubular filaments [91].

Specific tauopathies may display specific types of tau inclusions, as shown by argyrophilic grains and Pick's bodies. Pick's bodies are round and well-demarcated structures, normally occurring one per cell and constituted by heterogeneous types of tau filaments [92]. Unlike Pick's bodies, argyrophilic grains result positive for Gallyas staining and are characterized by a spindle shape mostly composed of straight tau filaments [93]. CBD/PSP and FTDP-17 on the other hand display pleomorphic neuronal tangle-type inclusions, which vary in size, shape and distribution [94-96]. Moreover, in the majority of tauopathies glial inclusions, in the form of coiled bodies, are found in oligodendrocytes [97, 98], astrocytic tufts and astrocytic plaques [99].

A recurring theme in neurodegenerative diseases is the co-occurrence of protein inclusions composed of different proteins in the same patient $[68,100]$. For example, tau and $\alpha$-synuclein inclusions co-occur in multiple diseases including Lewy body variant of AD, DLB and PD with dementia $[101,102]$. Despite the fact that tau and $\alpha$-synuclein are distinct proteins that contribute to different disease-specific pathologies, multiple lines of evidence suggest that tau and $\alpha$-synuclein interact, modulate the aggregation of each other and coexist in pathological inclusions in human brain [103]. Apart from the potential gain-of-toxic-function relationship that feeds forward the aggregation of each other, tau and $\alpha$-synuclein may also overlap in their loss-of-function effects via a partially shared protein interactome [68]. Similar cross talk may occur between other disease-associated aggregating proteins, e.g., between tau and TDP-43 [104].

In $\mathrm{AD}$, autopsy studies have demonstrated that pathological $\mathrm{A} \beta$ and tau inclusions accumulate in stereotypical spatial patterns over the course of the disease with the ADrelated tauopathy occurring downstream of amyloid pathology [105]. Development of PET tracers specifically binding to the aggregated forms of $\mathrm{A} \beta$ and tau now allows a better understanding of the temporal relationship of the amyloid and neurofibrillary pathologies in the human brain. It seems that the level of tau deposition in the temporal lobe is more closely related to dementia status and can predict cognitive performance better than $A \beta$ deposition in any brain region [106].

\section{Spreading of tau pathology in brain}

It is well established that in $\mathrm{AD}$ the development of tau pathology occurs in a hierarchical pattern of accumulation starting from the layer II of entorhinal cortex (EC) and spreading toward cortical regions, as originally proposed by the findings of Heiko and Eva Braak [107]. Increased cerebrospinal fluid (CSF) levels of tau are associated with faster rate of cognitive decline and overall worse clinical outcome in $\mathrm{AD}[108,109]$. Accumulating evidence suggests that in most neurodegenerative diseases, inclusions of misfolded and pathological proteins spread along neuroanatomically connected areas of the brain in a way that remind the spread of pathological prion protein (PrP) [110]. It was previously thought that stage-wise progression of neurodegeneration would reflect a differential spatial and temporal vulnerability of different brain areas to protein misfolding pathology and toxicity, therefore explaining the anatomical progression of the pathological inclusions [111]. However, the concept that pathologically misfolded protein species, such as tau aggregates, can be released from a cell and internalized by a neighboring cell from the extracellular space in a prion-like manner [110] has provided a new way of approaching the progression of tauopathies and other related neurodegenerative diseases.

Tau is able to serve as a hub for its own aggregation and as a seed for further misfolding in a process called templated misfolding. Once aggregated, the pathological tau seeds can be transmitted from one cell to another propagating pathology from affected to healthy cells and to previously unaffected brain areas. In 2009, a pioneer study demonstrated that tau extracted from tau(P301S) transgenic mice, which carries one of the frontotemporal dementia tau mutations and develops tau inclusions, and injected into healthy mice that do not normally develop tau inclusions, lead to development of tau pathology [112]. The authors detected extensive distribution of phosphorylated, filamentous and insoluble tau by silver staining and antibody labeling in the areas near the injection, as well as in neighboring areas of the brain, indicating that tau pathology can be seeded and propagated. A similar pathological propagation phenomenon has been reported in a number of studies, for e.g., tau extracted from human AD brains $[113,114]$ and from mice exposed to traumatic brain injury (TBI) [115].

Both in vitro and in vivo studies of tau propagation have been facilitated with the use of pre-formed fibrils (PFF) of tau, originally developed in the laboratory of John Trojanowsky and Virginia Lee [116]. Experiments performed with tau PFFs, both in vitro and in vivo, have revealed that tau can propagate trans-synaptically from one neuron to another, and that the nearby network, synaptic contacts and neuronal activity modulate such propagation $[117,118]$, possibly bridging the physiological release of tau $[119,120]$ and the 
pathological one [121]. Recent studies, using novel PET tracers binding to PHF-tau allowing real-time visualization of tau pathology, also support the view that progression of tau pathology occurs in the brains of human AD patients $[122,123]$.

It should also be taken into account that despite tau being a cytosolic and microtubule-associated protein, a physiological role for secreted tau is possible. Some evidence suggests that neuronal activity can influence tau release at the synaptic terminal and that such release is not connected to propagation of tau or transfer of any type of pathology to neighboring neurons $[119,120]$. Moreover, despite the origin and the degree of propagation substantially differing in different diseases, it is plausible that some common mechanistic features underlie the spread of pathology in various neurodegenerative diseases with different characteristic aggregating proteins. Thus, there is a clear need for better understanding of the specific mechanisms that drive tau secretion and of the specific forms of secreted tau and their different pathogenicity.

\section{Cell-to-cell transfer of tau}

Despite the fact that tau is a microtubule-associated cytosolic protein, it is physiologically present outside the cells. While many aspects of tau secretion remain unknown, it seems that tau can escape from cells via multiple routes (Fig. 2). Tau is found in the CSF [124], and CSF of AD patients displays elevated levels of phosphorylated tau species that seem to

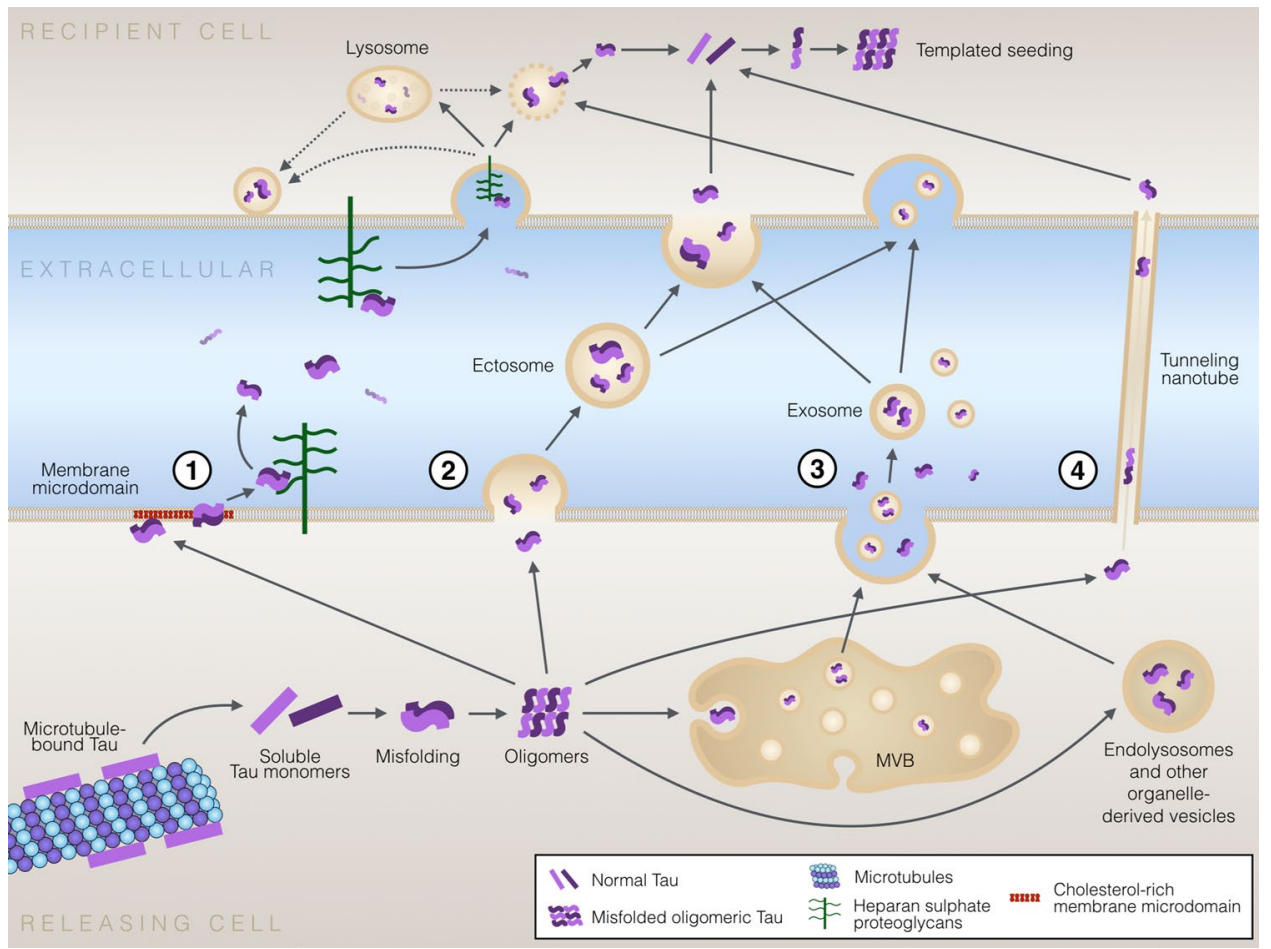

Fig. 2 Mechanisms of cell-to-cell transfer of pathological tau protein. Pathological tau conformers can be transferred between cells by multiple non-exclusive mechanisms. (1) Tau secretion directly through the PM involves clustering of tau at the PM, interaction with specific lipids in cholesterol/sphingomyelin/PI(4,5) $\mathrm{P}_{2}$-rich membrane microdomains, penetration through the PM and release from the PM facilitated by cell surface HSPGs. This unconventional tau secretion mechanism resembles the secretion of FGF2 (UPS I-like). (2) Tau is secreted in ectosomes shed from the PM. Ectosomes are larger than exosomes and also differ in their molecular composition. After their release from cells, both ectosomes and exosomes function similarly and can be fused to or endocytosed by target cells. (3) Secretion of tau in exosomes and via organelle hitchhiking. Tau can be packed in exosomes by inward budding of late endosome membrane leading to formation of intraluminal vesicles in multivesicular bodies (MVB) that can be secreted by fusion of MVB membrane with the PM. Other organelle hitch-hiking (UPS III-like) pathways possibly involved in secretion of tau and other misfolded cytosolic proteins include secretory endo-lysosomes, related to the autophagy-lysosomal pathway. The MAPS pathway promotes secretion of cytosolic misfolded proteins by chaperone-mediated capture of misfolded cytosolic proteins to the ER, followed by secretion via fusion of endo-lysosomal vesicles with the PM releasing vesicle-free tau in the extracellular space. (4) Cell-to-cell transfer of tau seeds via tunneling nanotubes that directly connect the cytosols of two neighboring cell. Regardless of the secretion pathway, tau aggregates eventually reach the cytosol of the recipient cells, allowing templated seeding of healthy tau molecules into misfolded pathological conformations. The recipient cells can then propagate the pathology further to other previously unaffected cells. It is currently unclear which ones of the above mechanisms are involved in synaptic release of tau, and whether the synaptic release of physiological and pathological forms of tau are mediated by the same mechanism(s) 
correlate with increased disease severity $[124,125]$. These findings suggest a prominent role for extracellular tau in disease pathogenesis and progression. Importantly, while a fraction of tau found in the cerebrospinal fluid may be associated with passive release of the intracellular content from dying cells, multiple lines of evidence suggest that active cellular processes are involved in tau secretion.

Some studies have shown that tau is secreted at the synaptic terminus during normal synaptic activity $[119,120]$. This constitutive and physiological secretion of tau has not been directly linked to propagation of pathological forms of tau to neighboring neurons, although the long-term effects of physiologically secreted tau are not known yet. It is also possible that secreted monomeric tau could have some yet unknown physiological signaling activity. Other studies, however, suggest that presynaptic neuronal activity may modulate the release and trans-synaptic transfer of pathological tau $[126,127]$. Furthermore, in animal models pathological tau appears to be more localized to synapses compared to non-pathological tau [63], and synaptosomes isolated from human AD brains were shown to contain more phosphorylated and aggregated tau than in healthy controls [64]. Although several studies have demonstrated that presynaptic neuronal activity enhances tau release, the mechanism of this phenomena, however, remains poorly understood. Neuronal depolarization or the associated presynaptic activity and $\mathrm{Ca}^{2+}$ response can modulate the release of two types of extracellular vesicles, ectosomes and exosomes [128, 129]. Indeed, Wang and colleagues have demonstrated that depolarization of neurons promotes the release of tau-containing exosomes [127]. Pooler et al., however, suggested that synaptic activity mainly modulates non-exosomal secretion of tau, through a mechanism dependent on the release of synaptic vesicles [119]. While it is still controversial how tau secretion occurs from synaptic terminals, whether associated with vesicles or via vesicle-free mechanism, it seems that in pathological conditions synapses are important for the spread of the pathogenic protein. Pathological spread of another misfolded protein, $\alpha$-synuclein, was recently shown to occur via the brain connectome [130] and it is plausible that similar neuronal network-mediated spread of tau pathology happens in tauopathies. This is supported by the evidence showing that the transmission of tau pathology occurs via a trans-synaptic mechanism, and that the pattern of spread is determined by synaptic connectivity rather than spatial proximity [131].

While in vivo studies have been critical for determining the cross-species seeding abilities of different pathological tau strains [132] and in demonstrating that pathological inclusions from human brains affected with tauopathies induce NFT formation in rodents, often recapitulating the disease-specific features of the original inclusion $[113,133$, 134], the molecular mechanisms underlying tau propagation remain challenging to assess in vivo. In vitro approaches have significantly contributed to our understanding of the basic mechanisms involved in the cell-to-cell transfer of tau pathology by, e.g., showing that morphologically distinct tau seeds can induce similar types of tau aggregates in recipient cells, surprisingly faithfully recapitulating morphological features of tau aggregates [74, 135]. Diverse molecular pathways that are involved in cell-to-cell transfer of pathological tau have been identified, drawing a heterogeneous landscape of mechanisms by which tau can exit cells and enter neighboring cells. Table 2 summarizes the currently available evidence supporting various cellular mechanisms involved in secretion of tau. There has been much focus on vesicular mechanisms, including exosomal secretion and release in PM-shed ectosomes, but also reports on vesicle-free secretion resulting in naked vesicle-free tau in the extracellular space. Different mechanisms are also involved in cellular uptake of vesicular tau and vesicle-free tau, and this likely affects the efficiency of cellular uptake, subcellular localization of internalized tau and the overall seeding potency of different forms of tau and in different cell types.

In addition to transfer of tau between cells via the extracellular space, a direct intercellular mechanism via tunneling nanotubes allows transport of cytosolic tau directly from the cytoplasm of one cell to the cytoplasm of another cell [136] (Fig. 2). Tunneling nanotubes, up to $100-\mu \mathrm{m}$ long communication paths between cells [137], have been shown to deliver pathological protein aggregates, including $\operatorname{PrP}, \alpha$-synuclein, tau, huntingtin and $\mathrm{A} \beta$, in naked form or associated with lysosomes [138]. Interestingly, tunneling nanotubes can mediate the transfer of pathological proteins not only between two neuronal cells, but between astrocytes as well [139]. Moreover, intracellular misfolded tau has been shown to promote the formation of tunneling nanotubes [140], possibly as an attempt to clear the cytoplasm from toxic proteins that cannot be otherwise cleared. However, the formation of tunneling nanotubes - or cellular contacts in other forms-is not required for cell-to-cell transfer of tau oligomers and aggregates, as several studies have shown that tau pathology can be transferred from one cell to another via the extracellular medium without cell-to-cell contacts or presence of donor cells $[118,121]$.

\section{Vesicle-mediated mechanisms of tau secretion}

Cellular protein secretion mechanisms are classified into conventional and unconventional. Conventional secretion mechanisms include the classical secretory route, also known as endoplasmic reticulum (ER)-Golgi pathway, for proteins that carry a signal peptide, which directs them to the ER membrane [141]. A vast majority of secreted proteins use the classical secretory route. It is estimated that $39 \%$ of the human protein-coding genes will produce one or 


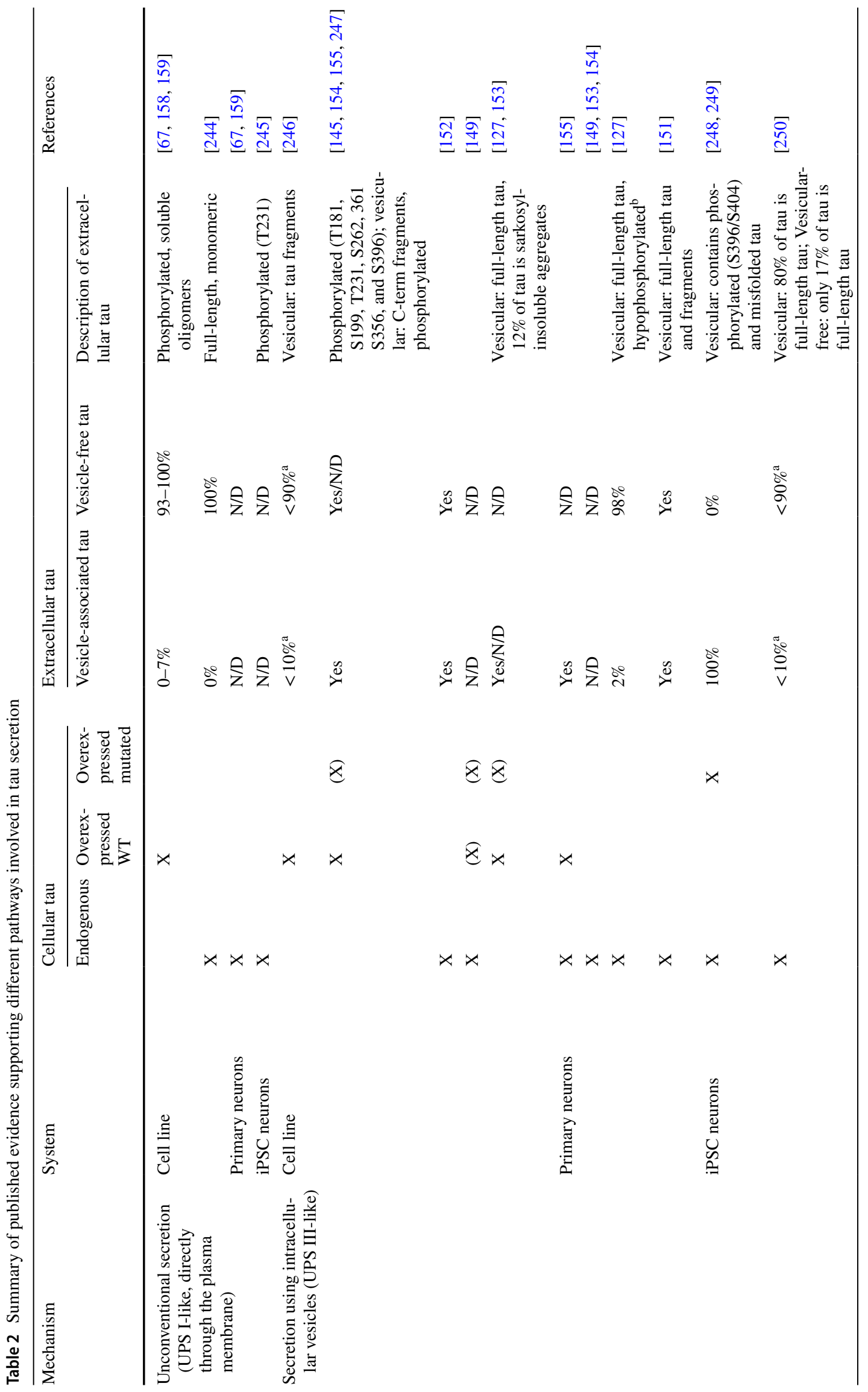




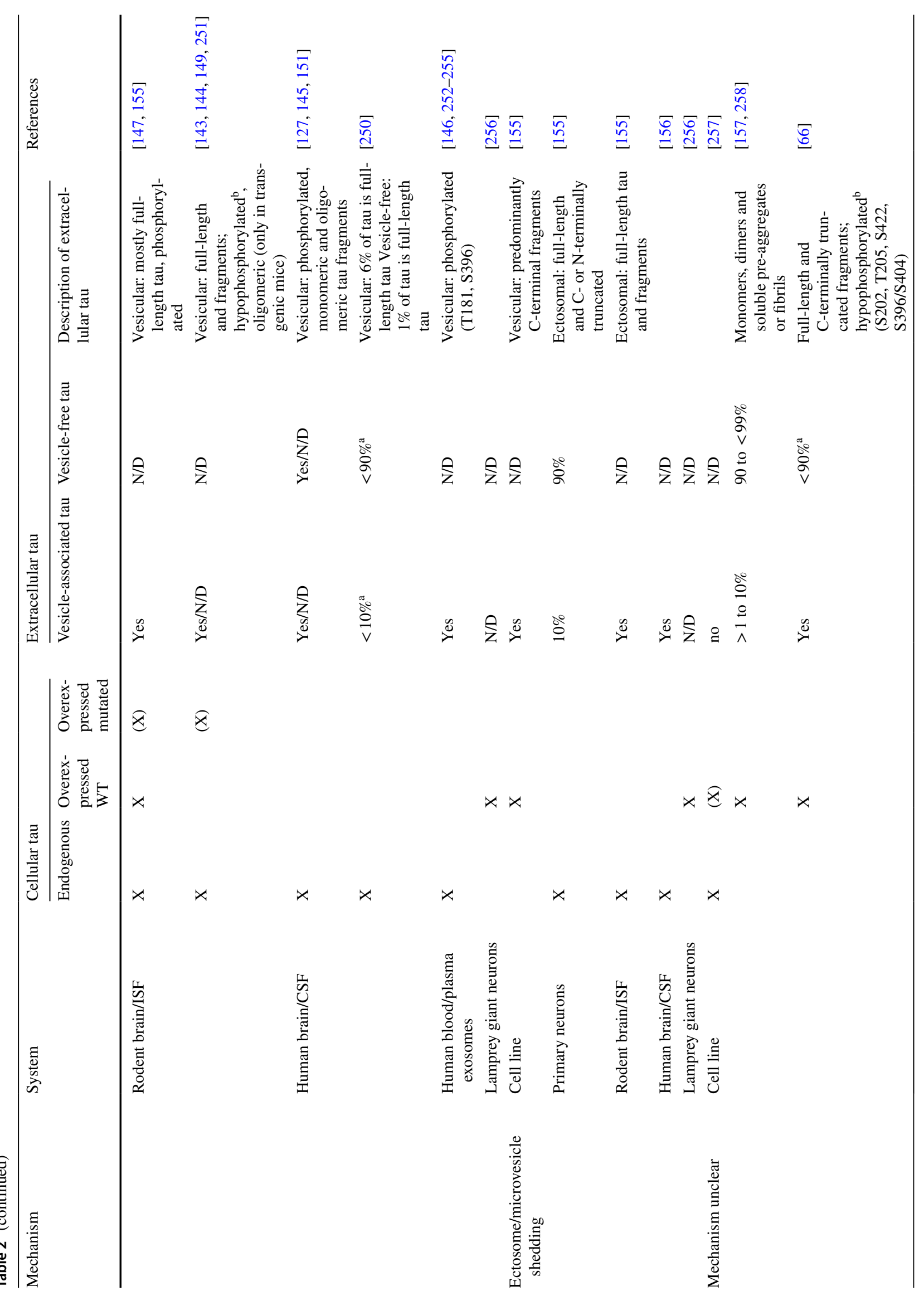




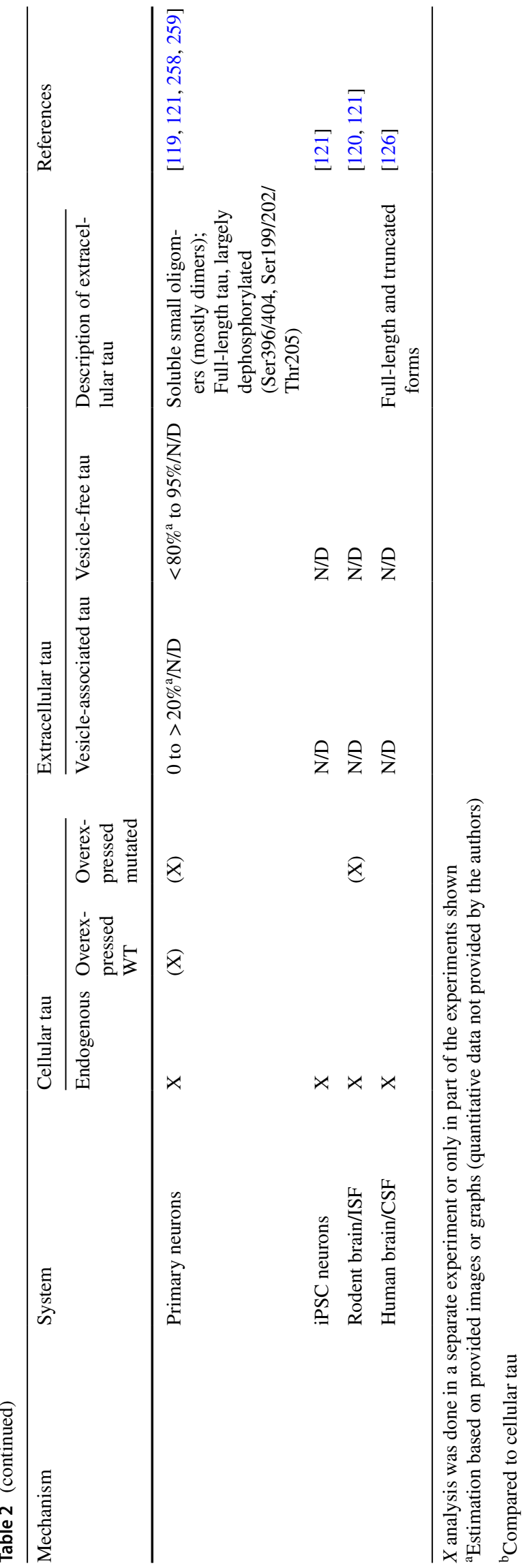

more products with a signal peptide, and $15 \%$ will produce a conventionally secreted protein product (proteinatlas.org). Unconventional secretion covers all the other routes, from PM pore formation [unconventional protein secretion type I (UPS I)] to organelle hitchhiking and exosomal secretion [unconventional protein secretion type III (UPS III)] and Golgi bypass [unconventional protein secretion type IV (UPS IV)] [142]. Unconventional protein secretion pathways are in most cases induced by cellular stress and are thus an important cellular survival strategy under proteostatic stress.

Different secretion mechanisms involving vesicular structures have been suggested for tau. One mechanism is UPS III-like and relies on intracellular structures, such as multivesicular bodies or endo-lysosomes, and may result in the secretion of tau inside small extracellular vesicles ( $\sim 0$ to $120 \mathrm{~nm}$ in diameter) called exosomes, but also in release of free tau, unbound to vesicles, through the fusion of endo-lysosomes with PM. Another mechanism, called microvesicle shedding, does not involve intracellular vesicular structures. The tau-containing vesicles are formed directly by outward budding of the PM, resulting in the secretion of tau in larger extracellular vesicles ( 100 to $1000 \mathrm{~nm}$ in diameter) called ectosomes.

Exosomes are generated in multivesicular bodies, a type of late endosomes, following inward budding of the outer endosomal membrane. Tau has been reported to be found in exosomes purified from brains of tau(P301L) transgenic mice $[143,144]$ and in exosomes isolated from neuroblastoma cells and from cerebrospinal fluid of AD patients [145]. Importantly, exosomes may deliver pathological tau to unaffected cells, seeding further tau pathology. Neuronderived exosomes extracted from plasma of mild cognitive impairment $(\mathrm{MCI})$ and $\mathrm{AD}$ patients are able to promote tau pathology in wild-type mice [146]. Finally, cell-to-cell transfer of tau via exosomes can be facilitated by microglia, whose role in prion-like propagation of protein aggregates remains incompletely understood [147].

Besides multivesicular bodies, tau may also be found in endo-lysosomes. Fusion of endo-lysosomes with the PM releases tau and other proteins to the extracellular space in a vesicle-free form [148]. Tau may be delivered to endolysosomes by multiple protein trafficking pathways. First, during cellular stress and overloading of the ubiquitin-proteasome clearing system, a misfolding-associated protein secretion pathway (MAPS) can be activated to translocate misfolded proteins, including tau, to endo-lysosomes for secretion to the extracellular space [148, 149]. The MAPS pathway involves the initial capture of misfolded proteins by the chaperone ubiquitin carboxyl-terminal hydrolase 19 (USP19) at the extracellular surface of ER. Then, co-chaperones Hsc70 and DNAJC5 mediate the sorting of misfolded cargos into the lumen of endo-lysosomes [148, 150]. Alternatively, dysfunctional cytoplasmic proteins and protein 
aggregates can be delivered to endo-lysosomes through macroautophagy [148]. Several reports have suggested a role of macroautophagy in tau secretion [151-154]. It is not clear, however, if autophagosomes deliver tau to endo-lysosomes for secretion or if they are capable of directly fusing to the PM for cargo release.

Ectosomes are larger than exosomes and are generated by outward budding of the PM rather than originating from intracellular multivesicular bodies [155]. Tau has been found in ectosomes purified from neuroblastoma cells, primary cortical neurons, mouse brain interstitial fluid (ISF) [155], and from cerebrospinal fluid of AD patients and healthy controls [156]. Interestingly, tau secretion in ectosomes was suggested to be a normal physiological phenomenon, while exosomal tau secretion may prevail under pathological conditions [155, 156].

It thus appears that tau utilizes different non-exclusive routes to overcome cellular membrane barriers and to exit the cell. It seems plausible that under proteostatic stress, the cells would activate multiple unconventional secretion pathways to clear the cytoplasm from the misfolded and aggregated proteins which endanger cellular survival. It is not clear yet whether different conformational tau species have preferred release pathways, and whether the pathogenicity of different conformers depends on them.

It is important to note that many of the studies that have reported secretion of tau in exosomes or ectosomes also demonstrated that a significant fraction of extracellular tau appears to be in the vesicle-free form. Additionally, some studies have described the secretion mechanisms utilized by tau independent of vesicular secretion (Table 2 and discussed below). The high relative level of vesicle-free tau compared to tau found in extracellular vesicles could be explained by three scenarios: (1) tau may be secreted in a free, non-vesicle-associated form, with a minority secreted in vesicles, (2) tau may be secreted exclusively in vesicles, but rapidly escaping most of the vesicles, or (3) tau may be secreted exclusively in free form, but may associate with extracellular vesicles after secretion. While most likely these scenarios are not mutually exclusive, increasing body of evidence suggests that tau release to the extracellular space may occur via unconventional secretion directly through the PM.

\section{Tau secretion directly through the plasma membrane}

Several studies suggest that while a fraction of the total extracellular tau is indeed found in exosomes or ectosomes, a large fraction of the extracellular tau pool exists in vesicle-free form $[149,155,157,158]$ (Table 2). Two recent studies demonstrated that tau secretion can occur via a type I mechanism of unconventional protein secretion (UPS I), i.e., by direct translocation across the PM [67,
159]. Using complementary methodological approaches, both groups concluded that direct secretion of tau through the PM involves the following key steps: (1) recruitment and clustering of tau at the cytosolic leaflet of the PM involving hyperphosphorylation of tau and specific lipids such as phosphatidyl inositol 4,5 phosphate $\left(\mathrm{PI}(4,5) \mathrm{P}_{2}\right)$ cholesterol and sphingolipids, and (2) release from the PM facilitated by binding to heparan sulfate proteoglycans (HSPG) located at the extracellular leaflet of the PM (Fig. 2). Although neither paper presented direct evidence of membrane pore formation, Katsinelos and colleagues showed that tau was capable of disrupting large unilamellar vesicle (LUV) membranes in a $\mathrm{PI}(4,5) \mathrm{P}_{2}$-dependent manner [67]. Interestingly, many of these mechanistic aspects of tau secretion have significant similarity to the unconventional secretion of fibroblast growth factor 2 (FGF2), one of the most widely studied unconventionally secreted proteins [160].

Tau localizes to the PM in a clustered manner, and this localization is enhanced by overexpression or hyperphosphorylation, possibly as a consequence of increased levels of cytosolic tau or enhanced aggregation. Transmission electron microscopy revealed two types of tau clusters, with diameter ranging between 50 and $200 \mathrm{~nm}$, at or very close to the PM [159]. Similar, reversible cation-sensitive clustering of tau has been previously reported for recombinant human tau in supported brain lipid membranes [161]. Experiments with large unilamellar vesicles (LUVs) demonstrated that tau and particularly its phosphomimetic mutant or hyperphosphorylated forms are able to bind to $\mathrm{PI}(4,5) \mathrm{P}_{2}$ and this interaction may be required for membrane binding of tau [67]. Several groups have shown that phosphorylation promotes tau secretion through the PM $[66,67]$, although one study correlates tau recruitment to the PM with hypophosphorylated states [162]. While FGF2 secretion requires specific phosphorylation by the Tec kinase at Y82, tau secretion seems to depend on the general level of phosphorylation rather than phosphorylation of a specific amino acid residue $[67,163,164]$. Plasma membrane localization and secretion of phosphomimetic mutant of tau were enhanced compared to wild-type tau [67]. Furthermore, the phospho-specific tau antibodies AT8 (Ser202/Thr205) and PHF13 (Ser396) stained mostly tau at or near the PM in neuroblastoma cells overexpressing tau [159]. Other post-translational modifications may also be involved in the secretion process. The two cysteine residues, located in the R2 and R3, may play a role in tau secretion as suggested by findings that at least some secreted tau species are disulfide bridged [165]. FGF2 secretion also involves intermolecular disulfide bridge formation, which may serve as a driver for FGF2 oligomerization at the membrane [166]. While there is no known mechanistic or pathophysiologic link between tau and FGF2, the similarities in their secretion mechanisms raises the possibility that tau could obtain a specific conformational state which 
may be able to engage an FGF2-like unconventional protein secretion mechanism at the PM. More broadly, this highlights the importance of UPS in understanding how pathological proteins may be released from cells.

To escape cells directly through the PM (or other membranes), tau would have to be able to interact with the membrane lipids. Indeed, tau interacts with both biological [13, 162, 167-170] and artificial membranes [17, 50, 171-175]. Binding of tau to membranes in vitro is enhanced by the presence of anionic lipids such as phosphatidyl serine (PS) and $\mathrm{PI}(4,5) \mathrm{P}_{2}[17,67,172,175]$. In a cellular context, tau may also require the presence of cholesterol and sphingomyelin for membrane interaction and penetration, indicating that cholesterol and sphingomyelin-dependent membrane microdomains (also called "lipid rafts") may play a role in this process $[18,159,176]$. Interestingly, tau may also disrupt ordered membrane domains and may thus affect the function of the PM more broadly [173].

Binding of tau to membranes has several important, related consequences that may affect its physiological and pathological functions and its secretion process. Firstly, tau can deform membranes in vitro. Tau can induce micellarization of anionic fatty acids [177] and deform micelles [17]. When tau was incubated with vesicles, tau-lipid complex formation resulted in morphological deformation of the vesicles [175]. Importantly, while individual MTBDs of tau can induce vesicle lysis [174]), full-length tau does not seem to share this activity, so tau membrane interaction does not seem to cause cell death per se [172]. To date, however, no biological consequences for this membrane deformation have been directly shown. Second, tau clusters [159, 161] and fibrillizes upon binding to anionic lipid membranes [49, 172, 177]. Tau fibrillization requires the presence of negatively charged nucleator, and anionic lipids in membranes can serve such a function. The inner leaflet of the PM of animal cells is composed of about $20 \%$ of anionic lipids that provide negative charges and create an electrostatic field for the inner leaflet. Thus, anionic membranes located at the inner leaflet of the PM can recruit tau to the PM and nucleate tau fibrillization. Third, tau, a natively unstructured protein acquires $\alpha$-helical conformation upon binding to membranes. The MTBDs of tau, especially R2 and R3, have been shown to undergo context-dependent folding on lipid membranes [17, 171, 175, 178]. The forming $\alpha$-helices are amphipathic, and mutating key hydrophobic residues in the helices inhibit binding of tau to membranes [175] and may therefore be important in tau secretion. These helices do not penetrate deeply into the lipid bilayer, but could act as molecular tweezers to extract negatively charged phospholipids from the membrane [17, 175]. Similar short $\alpha$-helices are also formed upon tau binding to microtubules [179], indicating that context-dependent folding of tau may take place with several interaction partners. Tau interaction with PM may also be partially mediated by interaction of tau N-terminus to PM-located proteins, such as annexin A2 and annexin A6 [180]. While membrane localization, deformation and membrane-dependent folding (and secretion) of tau could be important for the pathological forms of tau, it remains unclear if the lipid and membrane interactions play a role in physiological functions of tau in healthy cells.

A critical step in FGF2 secretion is the formation of a membrane pore by membrane-penetrating FGF2 oligomers. Tau was shown to disrupt membranes by forming pore-like amyloid structures [181, 182]. Importantly, posttranslational modifications and pathological mutations may enhance this property $[181,182]$. Tau aggregation/ oligomerization inhibitors significantly suppressed secretion of human tau overexpressed in cultured cells as well as endogenous tau from murine primary neurons [159]. While direct evidence of formation of membrane pores during the secretion process is lacking, these findings suggest that tau could penetrate the PM through an oligomerization-mediated process, somewhat similarly to FGF2. Interestingly, other disease-associated proteins, such as $\alpha$-synuclein and $\mathrm{A} \beta$, also have the ability to form bacteriotoxin-like annular protofibrils and pore-like structures in membranes [183, 184], suggesting that this mechanism could play a central role in unconventional secretion of several misfolded aggregating proteins capable of transcellular propagation. Importantly, membrane disruption by protofibrils could also provide an escape route from vesicles, both during the secretion process and after cellular uptake.

Lastly, like FGF2, tau secretion was also shown to depend on HSPG on cell surface to complete the translocation across the PM $[67,159]$. Tau has long been known to bind to GAGs in vitro, and heparin is widely used as a promoter of tau aggregation $[185,186]$. Tau secretion was efficiently suppressed when the cellular level of glycosaminoglycans (GAGs) was decreased by treatment with either sodium chlorate, a compound that inhibits sulfation of newly synthesized GAGs, or with heparinases, enzymes that specifically cleave heparin and heparan sulfate-type sulfated glycans [67, 159]. Chondroitinase $\mathrm{ABC}$ did not alter tau secretion indicating that HSPGs, but not chondroitin sulfate proteoglycans, are involved in the tau secretion process. Furthermore, tau secretion was impaired in $\mathrm{CHO}_{745}$ cells, which are deficient in the synthesis of proteoglycans due to lack of xylosyl transferase activity, but could be returned to almost normal level when wild-type cells were added to the culture, suggesting that even the presence of GAGs on neighboring cells is sufficient to facilitate tau secretion [67]. Importantly, sodium chlorate was able to reduce the secretion of endogenous tau from primary neurons $[67,159]$ suggesting that physiological secretion of tau may also involve GAGs. Altogether, these data suggest an important role for cell surface proteoglycans, specifically HSPGs, in tau secretion. 


\section{Cellular uptake and templated misfolding of tau in recipient cells}

After being secreted to the extracellular space, pathological tau oligomers, monomers or aggregates need to enter another cell via a process called cellular uptake. Following cellular uptake, pathological tau seeds may be degraded, re-secreted or can mediate the misfolding of healthy tau molecules in the recipient cells (Fig. 2). As for secretion, diverse cellular mechanisms can mediate tau internalization from the extracellular space. Recipient cells seem to favor low molecular weight, short tau fibrils over tau monomers and larger filaments [187], which may at least partially determine the potency of various tau aggregate species for cellular propagation [188]. Importantly, uptake of tau aggregates is not specific for neurons, and cell-to-cell transfer is likely to occur also between neurons and glial cells. Close proximity of the PMs of the donor and recipient cells, as is the case in synapses, appears to facilitate cell-to-cell transfer of tau aggregates [118].

Tau oligomers can be internalized by dynamin-dependent bulk endocytosis and transported within recipient cells in the endo-lysosomal pathway [187]. Micropinocytosis, another type of bulk and non-specific endocytosis, seems to play a central role in pathological tau uptake both in vitro and in vivo $[189,190]$, as well as for other pathologically misfolded proteins such as $\alpha$-synuclein, TDP-43 [191], and PrP [192]. Before being internalized by micropinocytosis, tau binds to HSPGs on the PM, which promote the rearrangements of the membrane prior to endocytosis [189]. Binding of tau to HSPGs appears to be crucial for internalization to occur, and 6- $O$-sulfation pattern of the heparan sulfate sidechains was reported to be a critical determinant for tau binding [193]. Interestingly, heparin-like GAG mimetics can mask the HSPG binding site on tau, resulting in reduced cell surface binding, uptake and seeding of tau oligomers [189].

HSPG-dependent macropinocytosis is initiated by small protein aggregates, and tau trimers were shown to be the minimum size to initiate this mechanism [193, 194]. In line with this, a recent study demonstrated that different tau species can be internalized by different cellular mechanisms [195]. Macropinocytosis is the preferred entry route for tau monomers and small oligomers (although they can also be internalized by endocytosis), while dynamin-dependent endocytosis is favoured for bigger aggregates.

Macropinocytosis and HSPGs may also be responsible for the uptake of whole exosomes [196], although it is possible that exosomes are internalized as a result of the unspecificity of micropinocytosis. In general, endocytosis and/or pinocytosis has been reported to be a favored internalization route for exosomes over direct fusion to the PM [197, 198].

Specific interactions with cell surface receptors may be involved in internalization and toxicity of tau oligomers. For example, tau interacts with muscarinic receptors, which may mediate tau internalization via clathrin-mediated endocytosis and contribute to tau toxicity by disrupting calcium homeostasis [199]. Blockage of muscarinic receptors indeed attenuates tau-mediated neurotoxicity in vitro [20].

Once tau is internalized from the extracellular space into the endosomes, it must find a way to escape the endosomal vesicles to interact with and template misfolding of cytosolic healthy tau molecules and to fully propagate the pathology to the recipient cells. Tau is indeed capable of rupturing the endosome membrane [200, 201], although the molecular mechanisms of this process, or which forms of tau mediate it, have not been fully elucidated. Organized tau conformations such as annular protofibrils and pore-forming structures [181, 182] could be involved in mediating tau escape from endo-lysosomal vesicles by disrupting the membrane in a manner recalling the escape of viruses or bacteria from endosomes [202]. Interestingly, C-terminal fragments of taucontaining MTBDs may be particularly effective in lysing vesicles $[172,174]$.

The concept of transcellular propagation of misfolding pathology implies that a pathological protein aggregate originating from a single or a restricted group of cells is able to promote pathology broadly to different brain areas in a way that the further misfolded and aggregated molecules resemble in morphology and pathogenicity of the original seed. Accumulating evidence demonstrates the ability of pathological tau to be internalized and to drive the conversion of healthy tau molecules into pathological aggregates, both in vitro and in vivo [112, 118, 133, 188]. Moreover, the morphological features of the original tau seeds can be faithfully recapitulated in the newly formed tau aggregates [74, 135]. Moreover, specific monomeric conformers have been shown to have different intrinsic abilities to become pathogenic, and they can give rise to a limited amount of strains, suggesting that the misfolding is closely related to the structure of the original seed [40,41].

Although mechanistic data are slowly emerging, it still remains unclear how the templated misfolding and seeding occurs at the molecular level. Two models have been proposed to explain the template seeding of endogenous tau. In the first model, called template-assisted growth, pathological oligomers act as templates for unfolded monomers, which are progressively packed into $\beta$-sheet structures and stabilized by hydrogen bonds [203, 204]. This model could explain the rapid process of tau fibrillization in vitro. In the second model, shared with $\operatorname{PrP}$ and $A \beta$ [205] and called oligomer-nucleated conformational induction, monomers are not directly assembled into bigger aggregates, but they oligomerize before being incorporated into fibrils [206]. Unfolded tau monomers are stabilized to the highest energy state to constitute the seed of rearranged proteins, which then accelerates the assembly process [206, 207]. 
However, the pathway of tau fibrillization may not be as straightforward as it seems. A recent study demonstrated that different stable monomeric forms of tau with different seeding abilities coexist, implying that different seeds could originate from their aggregation [40]. This study also raises the possibility that tau, despite being natively unfolded, may physiologically exist in relatively stable conformations that affect its propensity to adopt a pathological form. Additionally, the complex pattern of tau post-translational modifications may also contribute to generation of different conformers. Different tau pools might have slightly different aggregation properties leading to formation of different pathological aggregates of the same protein, with distinct structural composition, shape, toxicity and ultimately different disease specificity [74].

It is also important to note that, similarly to other disease-associated low complexity proteins [208-210], tau can undergo liquid-liquid phase separation, where tau separates into liquid-like droplets similarly as in a water-oil demixing event $[58,211]$. This process is mediated by the MTBDs and by electrostatic interactions between the negatively charged $\mathrm{N}$-terminus and positively charged C-terminus [58, 212]. Liquid-liquid phase separation spatially concentrates tau molecules, possibly favoring tau homo-oligomerization and aggregation. The concept that liquid-liquid phase separation may be transformed into permanent aggregation is now validated for several RNA-binding proteins associated with different neuropathological conditions [213]. Interestingly, TIA-1, an RNA-binding protein centrally involved in the formation of stress granules [214], has been shown to directly modulate tau pathology in vitro and in vivo [215, 216]. Interestingly, TIA-1-dependent recruitment of internalized tau oligomers to stress granules has been suggested to provide a hub for tau toxicity and seeding in recipient cells [190].

\section{Further considerations for understanding propagation of tau pathology in brain tissue}

Microglia play important and diverse roles in neurodegenerative diseases. Microglia have beneficial functions, such as clearing cellular debris, protein aggregates and dying cells, but can also contribute to disease pathogenesis by, e.g., abnormal synapse elimination and chronic pro-inflammatory cytokine secretion [217]. Post-mortem studies with patients affected by different tauopathies revealed deposits of aggregated tau in reactive microglia [218]. As microglia do not normally express tau, this indicates that microglia had engulfed tau aggregates from the extracellular space, or as a part of engulfed debris from dead neurons. It has been shown that microglia have the ability to internalize tau to degrade it [219-221]. However, when the amount of aggregating protein surpasses the degradation capabilities of microglia, toxic tau aggregates appear in the cytoplasm and promote microglial dysfunction [222]. While a correlation between microglia activation and spreading of tau pathology has been reported [223], the actual role of microglia in actively transferring pathological tau to neurons, and importantly also which forms of pathological tau are transferred, has been unclear. Importantly, microglia are able to promote cell-to-cell spreading of pathological tau by secreting it themselves [147]. In this study, microglia internalized neuronally secreted tau from the extracellular space and packed it in exosomes-which appear to contain the most toxic forms of tau [127, 155]. Consistently, when exosome formation was inhibited, tau propagation in neurons was reduced both in vitro and in vivo. Furthermore, depletion of microglia strongly suppressed propagation of tau pathology in human tau(P301S) expressing transgenic mice [147]. Additionally, astrocytes are also able to internalize both fibrillar and monomeric tau, implicating a possible role for other glial cells in the process of spreading the pathology [224, 225].

Factors affecting tau secretion or uptake, or efficiency of clearance of tau seeds from the brain interstitial fluid and CSF, likely affect the rate of disease progression in tauopathies. Genetic variants are known to contribute to the CSF levels of tau [226, 227]. However, currently it is not clear if and how genetic factors modulate individual susceptibility and rate of disease progression by altering propagation of tau pathology in the brain. Some mechanistic connections have been reported between late-onset AD risk gene function and tau secretion [157]. Moreover, expression and activities of several enzymes participating in generation and modification of sulfated glycosaminoglycans, particularly heparan sulfate sulfotransferases, have been reported to be altered in $\mathrm{AD}$ brain and to affect accumulation of phosphorylated tau species [228, 229]. While altered levels and function of HSPGs may relate to many aspects of AD pathogenesis, including ApoE function and growth factor activity in the brain, it is notable that HSPGs appear to play a key role in both secretion and uptake of pathological tau species.

One important contributor to clearance of tau aggregates from the brain is the brain glymphatic system, a brain-wide interstitial solute clearance system composed of astrocytes, paravascular routes and dural lymphatics [230, 231]. Clearance of extracellular tau from the brain was significantly impaired in mice lacking functional central nervous system lymphatic system [232]. Importantly, the glymphatic system function can be adversely affected by stroke, traumatic brain injury or other comorbidities, such as type 2 diabetes, which could enhance spreading of tau pathology in the brain [233, 234]. Similar to brain levels of $\mathrm{A} \beta$, sleep-wake cycle regulates brain ISF and CSF levels of tau in humans [235]. In P301S transgenic mice, increased wakefulness and sleep disruption increased ISF and CSF tau levels, tau aggregation and spreading over longer 
periods of time [236], suggesting that alterations in tau clearance can have a strong impact on progression of tau pathology.

There appears to be an intriguing connection between neuronal activity and tau secretion. Increased neuronal activity stimulates tau release in cultured cortical neurons [119], in human tau-overexpressing transgenic mice in vivo [120] and in human iPSC neurons [121]. Moreover, enhanced neuronal activity accelerates development and spreading of tau pathology in the brains of $\mathrm{rTg} 4510$ mice [121]. These data implicate that not only the pathological forms of tau, but also physiological "healthy" forms of tau are secreted by neurons in a manner that is regulated by neuronal activity. Currently, it is unclear which of the mechanisms involved in secretion of pathological forms of tau are also utilized in neuronal release of nonpathological forms of tau. HSPGs appear to be at least partially involved also in secretion of endogenous non-pathological forms of tau in cultured rodent neurons [67, 159]. Whether the neuronally secreted tau species are pathological and can seed tau pathology in neighboring cells could simply be determined by their conformational state. Somewhat unexpectedly, even tau monomers may carry such conformational information, as suggested by a recent study [41].

The findings that hyperexcitability promotes tau secretion may have important implications in the progression of tau pathology in $\mathrm{AD}$ patients. $\mathrm{A} \beta$ is known to promote hyperexcitability and subclinical epileptiform activity may contribute to cognitive decline in $\mathrm{AD}[237,238]$. It is possible that in regions vulnerable to early tauopathy, such as the hippocampus, amyloid pathology could trigger or promote the release and propagation of pathogenic tau seeds. In rTg4510 mice, optogenetic stimulation of the hippocampus leads to robust worsening of tau pathology [121], providing some experimental support for this type of mechanism.

Finally, all studies addressing tau secretion mechanisms should be performed thorough characterization of the secreted tau species, vesicular and non-vesicular, to improve comparability and interpretation of data from different sources. Another factor complicating comparison of different tau secretion studies is that some studies use full-length tau protein, while others use tau fragments, typically containing one or more MTBDs, yet the conclusions from most studies are generalized to tau (implicating the full-length tau protein). The diversity of tau arises from multiple levels, including splicing, conformational states, morphology of aggregates and filaments, and how this diversity is connected to transcellular propagation of tau is only beginning to be addressed.

\section{Concluding remarks}

The presence of tau in the cerebrospinal fluid of AD patients was long considered a mere consequence of passive release from degenerating and dying neurons. Accumulating evidence over the last decade has convincingly shown that tau and other neurodegeneration-associated intracellular amyloid proteins, such as $\alpha$-synuclein, TDP-43 and SOD1, can be actively secreted from cells and that these extracellular protein aggregates, or seeds, can propagate the misfolding pathology to healthy cells. It now seems that cell-to-cell transmission is a common mechanism for disease progression in most, if not all, neurodegenerative diseases.

Disruption of proteostasis can be particularly detrimental to neurons. Accumulation of misfolded aggregated proteins in cells triggers multiple cellular mechanisms that jointly aim to restore proteostasis and improve cell viability. Not surprisingly, pathological forms of tau can be secreted via multiple non-exclusive mechanisms, including both vesicular and non-vesicle-mediated pathways, some of which appear to be induced by accumulation of protein aggregates in cells. Also, unconventional protein secretion directly through the PM, similar to FGF2 secretion (UPS I-like), has recently been reported for tau. Many amyloid proteins have long been known to be membrane active, and formation of membrane pores may be involved in tau secretion but also in the escape of tau from vesicles, both in the extracellular space and after internalization to recipient cells. Although tau is considered a natively unfolded protein it appears to be capable of context-dependent folding, which could be important for both the aggregation process but also for the membrane interaction and secretion processes.

Tau aggregates can be diverse in terms of their morphology such as reflecting slightly different conformational states of the aggregated tau protein. It is currently unclear if particular secretion pathways are preferred by certain species of tau aggregates. It is noteworthy that recent studies suggest that even tau monomers may carry conformational information that can be replicated during the templated misfolding process, which can be propagated through generations of cells.

The fact that tau is physiologically secreted independently of aggregation raises the question if some forms of extracellular tau could have a yet undiscovered function in the nervous system. Whether physiological secretion of nonpathological forms of tau in neurons occurs via the same or overlapping mechanisms as the pathological forms of tau needs to be clarified in future studies. Importantly, both physiological and pathological secretion of tau appears to be connected to neuronal activity. The role of glial cells and the glymphatic system in the clearance and propagation of pathological protein aggregates deserves more attention in the future.

It is possible that cellular secretion and uptake mechanisms for the diverse range of disease-associated amyloid proteins are at least partially overlapping. Better understanding of the mechanisms of pathological protein propagation is expected to help develop novel therapeutic strategies for 
slowing down the progression of neurodegenerative diseases. For instance, the development of monoclonal antibodies or other compounds that specifically target, sequester or disassemble tau conformations involved in the propagation of pathology could slow down disease progression in tauopathies. Alternatively, inhibition of the processes that promote tau interaction with plasma membrane lipids or cell surface proteoglycans may decrease the extracellular pool of tau available for internalization by neighboring cells. Inhibition of the uptake of secreted tau species or interfering with the templated misfolding or stress granule association of tau in the recipient cells could provide another therapeutic strategy for halting transcellular propagation of tau pathology. However, mechanistically we should first aim at better understanding of the different roles and functions of physiological vs pathological forms of secreted tau and also clarify whether the same, overlapping and distinct secretion mechanisms are responsible for secretion of the two different pools of extracellular tau.

Acknowledgements Open access funding provided by University of Helsinki including Helsinki University Central Hospital.

Funding This work has been supported by grants from the Academy of Finland (Grant Number 296409) and Alfred Kordelin Foundation (RLU).

\section{Compliance with ethical standards}

Conflict of interest $\mathrm{HJH}$ is an employee and a shareholder of Herantis Pharma Plc, which is not related to the contents of this review.

Open Access This article is distributed under the terms of the Creative Commons Attribution 4.0 International License (http://creativeco mmons.org/licenses/by/4.0/), which permits unrestricted use, distribution, and reproduction in any medium, provided you give appropriate credit to the original author(s) and the source, provide a link to the Creative Commons license, and indicate if changes were made.

\section{References}

1. Aamodt EJ, Williams RC Jr (1984) Microtubule-associated proteins connect microtubules and neurofilaments in vitro. Biochemistry 23(25):6023-6031

2. Bertrand A et al (2013) Non-invasive, in vivo monitoring of neuronal transport impairment in a mouse model of tauopathy using MEMRI. Neuroimage 64:693-702

3. Seitz A, Kojima H, Oiwa K, Mandelkow EM, Song YH, Mandelkow E (2002) Single-molecule investigation of the interference between kinesin, tau and MAP2c. EMBO J 21(18):4896-4905

4. Zhang B et al (2004) Retarded axonal transport of R406W mutant tau in transgenic mice with a neurodegenerative tauopathy. $\mathrm{J}$ Neurosci 24(19):4657-4667

5. Ebneth A, Godemann R, Stamer K, Illenberger S, Trinczek B, Mandelkow E (1998) Overexpression of tau protein inhibits kinesin-dependent trafficking of vesicles, mitochondria, and endoplasmic reticulum: implications for Alzheimer's disease. J Cell Biol 143(3):777-794

6. Weingarten MD, Lockwood AH, Hwo SY, Kirschner MW (1975) A protein factor essential for microtubule assembly. Proc Natl Acad Sci USA 72(5):1858-1862

7. Muller R, Heinrich M, Heck S, Blohm D, Richter-Landsberg C (1997) Expression of microtubule-associated proteins MAP2 and tau in cultured rat brain oligodendrocytes. Cell Tissue Res 288(2):239-249

8. Camero S et al (2014) Tau protein provides DNA with thermodynamic and structural features which are similar to those found in histone-DNA complex. J Alzheimers Dis 39(3):649-660

9. Li XC et al (2016) Human wild-type full-length tau accumulation disrupts mitochondrial dynamics and the functions via increasing mitofusins. Sci Rep 6:24756

10. Ittner LM et al (2010) Dendritic function of tau mediates amyloid-beta toxicity in Alzheimer's disease mouse models. Cell 142(3):387-397

11. Kimura $\mathrm{T}$ et al (2014) Microtubule-associated protein tau is essential for long-term depression in the hippocampus. Philos Trans R Soc Lond B Biol Sci 369(1633):20130144

12. Dou $\mathrm{F}$ et al (2003) Chaperones increase association of tau protein with microtubules. Proc Natl Acad Sci USA 100(2):721-726

13. Brandt R, Leger J, Lee G (1995) Interaction of tau with the neural plasma membrane mediated by tau's amino-terminal projection domain. J Cell Biol 131(5):1327-1340

14. Pallas-Bazarra $\mathrm{N}$ et al (2016) Novel function of Tau in regulating the effects of external stimuli on adult hippocampal neurogenesis. EMBO J 35(13):1417-1436

15. Camero S, Benitez MJ, Cuadros R, Hernandez F, Avila J, Jimenez JS (2014) Thermodynamics of the interaction between Alzheimer's disease related tau protein and DNA. PLoS One 9(8): 104690

16. Sultan A et al (2011) Nuclear tau, a key player in neuronal DNA protection. J Biol Chem 286(6):4566-4575

17. Georgieva ER, Xiao S, Borbat PP, Freed JH, Eliezer D (2014) Tau binds to lipid membrane surfaces via short amphipathic helices located in its microtubule-binding repeats. Biophys $\mathrm{J}$ 107(6):1441-1452

18. Klein C, Kramer EM, Cardine AM, Schraven B, Brandt R, Trotter J (2002) Process outgrowth of oligodendrocytes is promoted by interaction of fyn kinase with the cytoskeletal protein tau. J Neurosci 22(3):698-707

19. Cardona-Gomez GP, Arango-Davila C, Gallego-Gomez JC, Barrera-Ocampo A, Pimienta H, Garcia-Segura LM (2006) Estrogen dissociates Tau and alpha-amino-3-hydroxy-5-methylisoxazole4-propionic acid receptor subunit in postischemic hippocampus. NeuroReport 17(12):1337-1341

20. Gomez-Ramos A, Diaz-Hernandez M, Rubio A, Miras-Portugal MT, Avila J (2008) Extracellular tau promotes intracellular calcium increase through M1 and M3 muscarinic receptors in neuronal cells. Mol Cell Neurosci 37(4):673-681

21. Goedert JJ et al (1989) A prospective study of human immunodeficiency virus type 1 infection and the development of AIDS in subjects with hemophilia. N Engl J Med 321(17):1141-1148

22. Guo T, Noble W, Hanger DP (2017) Roles of tau protein in health and disease. Acta Neuropathol 133(5):665-704

23. Matsumoto SE et al (2015) The twenty-four kDa C-terminal tau fragment increases with aging in tauopathy mice: implications of prion-like properties. Hum Mol Genet 24(22):6403-6416

24. Paholikova K et al (2015) N-terminal truncation of microtubule associated protein tau dysregulates its cellular localization. J Alzheimers Dis 43(3):915-926 
25. Gauthier-Kemper A et al (2011) The frontotemporal dementia mutation R406W blocks tau's interaction with the membrane in an annexin A2-dependent manner. J Cell Biol 192(4):647-661

26. Morris M, Maeda S, Vossel K, Mucke L (2011) The many faces of tau. Neuron 70(3):410-426

27. Wang $X$ et al (2006) The proline-rich domain and the microtubule binding domain of protein tau acting as RNA binding domains. Protein Pept Lett 13(7):679-685

28. Quintanilla RA, Matthews-Roberson TA, Dolan PJ, Johnson GV (2009) Caspase-cleaved tau expression induces mitochondrial dysfunction in immortalized cortical neurons: implications for the pathogenesis of Alzheimer disease. J Biol Chem 284(28): 18754-18766

29. Goedert M, Spillantini MG (2017) Propagation of Tau aggregates. Mol Brain 10(1):18

30. Ghetti B, Oblak AL, Boeve BF, Johnson KA, Dickerson BC, Goedert M (2015) Invited review: frontotemporal dementia caused by microtubule-associated protein tau gene (MAPT) mutations: a chameleon for neuropathology and neuroimaging. Neuropathol Appl Neurobiol 41(1):24-46

31. Hutton $\mathrm{M}$ et al (1998) Association of missense and 5'-splicesite mutations in tau with the inherited dementia FTDP-17. Nature 393(6686):702-705

32. Spillantini MG, Murrell JR, Goedert M, Farlow MR, Klug A, Ghetti B (1998) Mutation in the tau gene in familial multiple system tauopathy with presenile dementia. Proc Natl Acad Sci USA 95(13):7737-7741

33. Sposito $\mathrm{T}$ et al (2015) Developmental regulation of tau splicing is disrupted in stem cell-derived neurons from frontotemporal dementia patients with the $10+16$ splice-site mutation in MAPT. Hum Mol Genet 24(18):5260-5269

34. Liu F, Gong CX (2008) Tau exon 10 alternative splicing and tauopathies. Mol Neurodegener 3:8

35. Zhong Q, Congdon EE, Nagaraja HN, Kuret J (2012) Tau isoform composition influences rate and extent of filament formation. J Biol Chem 287(24):20711-20719

36. von Bergen M, Friedhoff P, Biernat J, Heberle J, Mandelkow EM, Mandelkow E (2000) Assembly of tau protein into Alzheimer paired helical filaments depends on a local sequence motif ((306)VQIVYK(311)) forming beta structure. Proc Natl Acad Sci USA 97(10):5129-5134

37. Simic G, Diana A, Hof PR (2003) Phosphorylation pattern of tau associated with distinct changes of the growth cone cytoskeleton. Prog Mol Subcell Biol 32:33-48

38. Cleveland DW, Hwo SY, Kirschner MW (1977) Physical and chemical properties of purified tau factor and the role of tau in microtubule assembly. J Mol Biol 116(2):227-247

39. Avila J et al (2016) Tau structures. Front Aging Neurosci 8:262

40. Mirbaha $\mathrm{H}$ et al (2018) Inert and seed-competent tau monomers suggest structural origins of aggregation. Elife 7:e36584

41. Sharma AM, Thomas TL, Woodard DR, Kashmer OM, Diamond MI (2018) Tau monomer encodes strains. Elife 7:e37813

42. Kellogg EH, Hejab NMA, Poepsel S, Downing KH, DiMaio F, Nogales E (2018) Near-atomic model of microtubule-tau interactions. Science 360(6394):1242-1246

43. Perez M, Valpuesta JM, Medina M, Montejo de Garcini E, Avila J (1996) Polymerization of tau into filaments in the presence of heparin: the minimal sequence required for tau-tau interaction. J Neurochem 67(3):1183-1190

44. Schweers O, Mandelkow EM, Biernat J, Mandelkow E (1995) Oxidation of cysteine-322 in the repeat domain of microtubuleassociated protein tau controls the in vitro assembly of paired helical filaments. Proc Natl Acad Sci USA 92(18):8463-8467

45. Mercken M, Grynspan F, Nixon RA (1995) Differential sensitivity to proteolysis by brain calpain of adult human tau, fetal human tau and PHF-tau. FEBS Lett 368(1):10-14
46. Fitzpatrick AWP et al (2017) Cryo-EM structures of tau filaments from Alzheimer's disease. Nature 547(7662):185-190

47. Kirschner DA, Abraham C, Selkoe DJ (1986) X-ray diffraction from intraneuronal paired helical filaments and extraneuronal amyloid fibers in Alzheimer disease indicates cross-beta conformation. Proc Natl Acad Sci USA 83(2):503-507

48. Bulic B, Pickhardt M, Mandelkow EM, Mandelkow E (2010) Tau protein and tau aggregation inhibitors. Neuropharmacology 59(4-5):276-289

49. King ME, Gamblin TC, Kuret J, Binder LI (2000) Differential assembly of human tau isoforms in the presence of arachidonic acid. J Neurochem 74(4):1749-1757

50. Kunze G, Barre P, Scheidt HA, Thomas L, Eliezer D, Huster D (2012) Binding of the three-repeat domain of tau to phospholipid membranes induces an aggregated-like state of the protein. Biochim Biophys Acta 1818(9):2302-2313

51. Kampers T, Friedhoff P, Biernat J, Mandelkow EM, Mandelkow E (1996) RNA stimulates aggregation of microtubule-associated protein tau into Alzheimer-like paired helical filaments. FEBS Lett 399(3):344-349

52. Ramachandran G, Udgaonkar JB (2011) Understanding the kinetic roles of the inducer heparin and of rod-like protofibrils during amyloid fibril formation by Tau protein. J Biol Chem 286(45):38948-38959

53. Goedert M, Jakes R (1990) Expression of separate isoforms of human tau protein: correlation with the tau pattern in brain and effects on tubulin polymerization. EMBO J 9(13):4225-4230

54. Ksiezak-Reding H, Liu WK, Yen SH (1992) Phosphate analysis and dephosphorylation of modified tau associated with paired helical filaments. Brain Res 597(2):209-219

55. Hanger DP, Anderton BH, Noble W (2009) Tau phosphorylation: the therapeutic challenge for neurodegenerative disease. Trends Mol Med 15(3):112-119

56. Grundke-Iqbal I, Iqbal K, Tung YC, Quinlan M, Wisniewski HM, Binder LI (1986) Abnormal phosphorylation of the microtubuleassociated protein tau (tau) in Alzheimer cytoskeletal pathology. Proc Natl Acad Sci USA 83(13):4913-4917

57. Alonso AD, Grundke-Iqbal I, Barra HS, Iqbal K (1997) Abnormal phosphorylation of tau and the mechanism of Alzheimer neurofibrillary degeneration: sequestration of microtubule-associated proteins 1 and 2 and the disassembly of microtubules by the abnormal tau. Proc Natl Acad Sci USA 94(1):298-303

58. Wegmann $\mathrm{S}$ et al (2018) Tau protein liquid-liquid phase separation can initiate tau aggregation. EMBO J 37(7):e98049

59. Ittner LM et al (2008) Parkinsonism and impaired axonal transport in a mouse model of frontotemporal dementia. Proc Natl Acad Sci USA 105(41):15997-16002

60. Di J, Cohen LS, Corbo CP, Phillips GR, El Idrissi A, Alonso AD (2016) Abnormal tau induces cognitive impairment through two different mechanisms: synaptic dysfunction and neuronal loss. Sci Rep 6:20833

61. Zhou L et al (2017) Tau association with synaptic vesicles causes presynaptic dysfunction. Nat Commun 8:15295

62. Hoover BR et al (2010) Tau mislocalization to dendritic spines mediates synaptic dysfunction independently of neurodegeneration. Neuron 68(6):1067-1081

63. Sahara N, Murayama M, Higuchi M, Suhara T, Takashima A (2014) Biochemical distribution of tau protein in synaptosomal fraction of transgenic mice expressing human P301L Tau. Front Neurol 5:26

64. Tai HC, Wang BY, Serrano-Pozo A, Frosch MP, Spires-Jones TL, Hyman BT (2014) Frequent and symmetric deposition of misfolded tau oligomers within presynaptic and postsynaptic terminals in Alzheimer's disease. Acta Neuropathol Commun $2: 146$ 
65. Dickey CA et al (2007) The high-affinity HSP90-CHIP complex recognizes and selectively degrades phosphorylated tau client proteins. J Clin Invest 117(3):648-658

66. Plouffe V, Mohamed NV, Rivest-McGraw J, Bertrand J, Lauzon M, Leclerc N (2012) Hyperphosphorylation and cleavage at D421 enhance tau secretion. PLoS One 7(5):e36873

67. Katsinelos $\mathrm{T}$ et al (2018) Unconventional secretion mediates the trans-cellular spreading of Tau. Cell Rep 23(7):2039-2055

68. Yan X, Uronen RL, Huttunen HJ (2018) The interaction of alphasynuclein and Tau: a molecular conspiracy in neurodegeneration? Semin Cell Dev Biol S1084-9521(17):30389

69. Martin L, Latypova X, Terro F (2011) Post-translational modifications of tau protein: implications for Alzheimer's disease. Neurochem Int 58(4):458-471

70. Liu F, Grundke-Iqbal I, Iqbal K, Gong CX (2005) Contributions of protein phosphatases PP1, PP2A, PP2B and PP5 to the regulation of tau phosphorylation. Eur J Neurosci 22(8):1942-1950

71. Wang Y, Mandelkow E (2016) Tau in physiology and pathology. Nat Rev Neurosci 17(1):5-21

72. Schneider A, Biernat J, von Bergen M, Mandelkow E, Mandelkow EM (1999) Phosphorylation that detaches tau protein from microtubules (Ser262, Ser214) also protects it against aggregation into Alzheimer paired helical filaments. Biochemistry 38(12):3549-3558

73. Arendt $\mathrm{T}$ et al (2003) Reversible paired helical filamentlike phosphorylation of tau is an adaptive process associated with neuronal plasticity in hibernating animals. J Neurosci 23(18):6972-6981

74. Sanders DW et al (2014) Distinct tau prion strains propagate in cells and mice and define different tauopathies. Neuron 82(6):1271-1288

75. Gotz J, Chen F, van Dorpe J, Nitsch RM (2001) Formation of neurofibrillary tangles in P3011 tau transgenic mice induced by Abeta 42 fibrils. Science 293(5534):1491-1495

76. Chabrier MA, Blurton-Jones M, Agazaryan AA, Nerhus JL, Martinez-Coria H, LaFerla FM (2012) Soluble abeta promotes wild-type tau pathology in vivo. J Neurosci 32(48):17345-17350

77. Oddo S, Caccamo A, Kitazawa M, Tseng BP, LaFerla FM (2003) Amyloid deposition precedes tangle formation in a triple transgenic model of Alzheimer's disease. Neurobiol Aging 24(8):1063-1070

78. Giasson BI et al (2003) Initiation and synergistic fibrillization of tau and alpha-synuclein. Science 300(5619):636-640

79. Ishizawa T, Mattila P, Davies P, Wang D, Dickson DW (2003) Colocalization of tau and alpha-synuclein epitopes in Lewy bodies. J Neuropathol Exp Neurol 62(4):389-397

80. Nalls MA et al (2014) Large-scale meta-analysis of genomewide association data identifies six new risk loci for Parkinson's disease. Nat Genet 46(9):989-993

81. Paul KC et al (2016) APOE, MAPT, and COMT and Parkinson's disease susceptibility and cognitive symptom progression. J Parkinsons Dis 6(2):349-359

82. Tolnay M, Clavaguera F (2004) Argyrophilic grain disease: a late-onset dementia with distinctive features among tauopathies. Neuropathology 24(4):269-283

83. Neary D et al (1998) Frontotemporal lobar degeneration: a consensus on clinical diagnostic criteria. Neurology 51(6):1546-1554

84. Kovacs GG (2015) Invited review: neuropathology of tauopathies: principles and practice. Neuropathol Appl Neurobiol 41(1):3-23

85. Iqbal K, Liu F, Gong CX, Grundke-Iqbal I (2010) Tau in Alzheimer disease and related tauopathies. Curr Alzheimer Res 7(8):656-664

86. Foster NL, Wilhelmsen K, Sima AA, Jones MZ, D'Amato CJ, Gilman S (1997) Frontotemporal dementia and parkinsonism linked to chromosome 17: a consensus conference. Conference participants. Ann Neurol 41(6):706-715

87. Forrest SL et al (2018) Retiring the term FTDP-17 as MAPT mutations are genetic forms of sporadic frontotemporal tauopathies. Brain 141(2):521-534

88. Hong $M$ et al (1998) Mutation-specific functional impairments in distinct tau isoforms of hereditary FTDP-17. Science 282(5395):1914-1917

89. Delacourte A, Sergeant N, Wattez A, Gauvreau D, Robitaille Y (1998) Vulnerable neuronal subsets in Alzheimer's and Pick's disease are distinguished by their tau isoform distribution and phosphorylation. Ann Neurol 43(2):193-204

90. Delisle MB et al (1999) A mutation at codon 279 (N279K) in exon 10 of the Tau gene causes a tauopathy with dementia and supranuclear palsy. Acta Neuropathol 98(1):62-77

91. Kovacs GG et al (2008) MAPT S305I mutation: implications for argyrophilic grain disease. Acta Neuropathol 116(1):103-118

92. Munoz-Garcia D, Ludwin SK (1984) Classic and generalized variants of Pick's disease: a clinicopathological, ultrastructural, and immunocytochemical comparative study. Ann Neurol 16(4):467-480

93. Braak H, Braak E (1989) Cortical and subcortical argyrophilic grains characterize a disease associated with adult onset dementia. Neuropathol Appl Neurobiol 15(1):13-26

94. Gibb WR, Luthert PJ, Marsden CD (1989) Corticobasal degeneration. Brain 112(Pt 5):1171-1192

95. Spillantini MG, Crowther RA, Kamphorst W, Heutink P, van Swieten JC (1998) Tau pathology in two Dutch families with mutations in the microtubule-binding region of tau. Am J Pathol 153(5):1359-1363

96. Dickson DW, Ahmed Z, Algom AA, Tsuboi Y, Josephs KA (2010) Neuropathology of variants of progressive supranuclear palsy. Curr Opin Neurol 23(4):394-400

97. Yamada T, McGeer PL (1990) Oligodendroglial microtubular masses: an abnormality observed in some human neurodegenerative diseases. Neurosci Lett 120(2):163-166

98. Dickson DW (1999) Neuropathologic differentiation of progressive supranuclear palsy and corticobasal degeneration. J Neurol 246(Suppl 2):II6-II15

99. Komori T et al (1998) Astrocytic plaques and tufts of abnormal fibers do not coexist in corticobasal degeneration and progressive supranuclear palsy. Acta Neuropathol 96(4):401-408

100. Takeda T (2018) Possible concurrence of TDP-43, tau and other proteins in amyotrophic lateral sclerosis/frontotemporal lobar degeneration. Neuropathology 38(1):72-81

101. Arima $\mathrm{K}$ et al (2000) NACP/alpha-synuclein and tau constitute two distinctive subsets of filaments in the same neuronal inclusions in brains from a family of parkinsonism and dementia with Lewy bodies: double-immunolabeling fluorescence and electron microscopic studies. Acta Neuropathol 100(2):115-121

102. Wills J, Jones J, Haggerty T, Duka V, Joyce JN, Sidhu A (2010) Elevated tauopathy and alpha-synuclein pathology in postmortem Parkinson's disease brains with and without dementia. Exp Neurol 225(1):210-218

103. Badiola $\mathrm{N}$ et al (2011) Tau enhances alpha-synuclein aggregation and toxicity in cellular models of synucleinopathy. PLoS One 6(10):e26609

104. Davis SA, Gan KA, Dowell JA, Cairns NJ, Gitcho MA (2017) TDP-43 expression influences amyloidbeta plaque deposition and tau aggregation. Neurobiol Dis 103:154-162

105. Jack CR Jr et al (2013) Tracking pathophysiological processes in Alzheimer's disease: an updated hypothetical model of dynamic biomarkers. Lancet Neurol 12(2):207-216

106. Brier MR et al (2016) Tau and Abeta imaging, CSF measures, and cognition in Alzheimer's disease. Sci Transl Med 8(338):338ra66 
107. Braak H, Braak E (1991) Neuropathological staging of Alzheimer-related changes. Acta Neuropathol 82(4):239-259

108. Wallin AK, Blennow K, Zetterberg H, Londos E, Minthon L, Hansson O (2010) CSF biomarkers predict a more malignant outcome in Alzheimer disease. Neurology 74(19):1531-1537

109. Kester MI et al (2009) CSF biomarkers predict rate of cognitive decline in Alzheimer disease. Neurology 73(17):1353-1358

110. Jucker M, Walker LC (2013) Self-propagation of pathogenic protein aggregates in neurodegenerative diseases. Nature 501(7465):45-51

111. Walsh DM, Selkoe DJ (2016) A critical appraisal of the pathogenic protein spread hypothesis of neurodegeneration. Nat Rev Neurosci 17(4):251-260

112. Clavaguera F et al (2009) Transmission and spreading of tauopathy in transgenic mouse brain. Nat Cell Biol 11(7):909-913

113. Clavaguera F et al (2013) Brain homogenates from human tauopathies induce tau inclusions in mouse brain. Proc Natl Acad Sci USA 110(23):9535-9540

114. Lasagna-Reeves CA et al (2012) Alzheimer brain-derived tau oligomers propagate pathology from endogenous tau. Sci Rep 2:700

115. Zanier ER et al (2018) Induction of a transmissible tau pathology by traumatic brain injury. Brain 141(9):2685-2699

116. Guo JL, Lee VM (2011) Seeding of normal Tau by pathological Tau conformers drives pathogenesis of Alzheimer-like tangles. J Biol Chem 286(17):15317-15331

117. Iba M, Guo JL, McBride JD, Zhang B, Trojanowski JQ, Lee VM (2013) Synthetic tau fibrils mediate transmission of neurofibrillary tangles in a transgenic mouse model of Alzheimer's-like tauopathy. J Neurosci 33(3):1024-1037

118. Calafate $\mathrm{S}$ et al (2015) Synaptic contacts enhance cell-to-cell tau pathology propagation. Cell Rep 11(8):1176-1183

119. Pooler AM, Phillips EC, Lau DH, Noble W, Hanger DP (2013) Physiological release of endogenous tau is stimulated by neuronal activity. EMBO Rep 14(4):389-394

120. Yamada K et al (2014) Neuronal activity regulates extracellular tau in vivo. J Exp Med 211(3):387-393

121. Wu JW et al (2016) Neuronal activity enhances tau propagation and tau pathology in vivo. Nat Neurosci 19(8):1085-1092

122. Schwarz AJ et al (2016) Regional profiles of the candidate tau PET ligand 18F-AV-1451 recapitulate key features of Braak histopathological stages. Brain 139(Pt 5):1539-1550

123. Lowe VJ et al (2018) Widespread brain tau and its association with ageing, Braak stage and Alzheimer's dementia. Brain 141(1):271-287

124. Arai $\mathrm{H}$ et al (1995) Tau in cerebrospinal fluid: a potential diagnostic marker in Alzheimer's disease. Ann Neurol 38(4):649-652

125. Hampel H, Blennow K, Shaw LM, Hoessler YC, Zetterberg H, Trojanowski JQ (2010) Total and phosphorylated tau protein as biological markers of Alzheimer's disease. Exp Gerontol 45(1):30-40

126. Sokolow $\mathrm{S}$ et al (2015) Pre-synaptic C-terminal truncated tau is released from cortical synapses in Alzheimer's disease. J Neurochem 133(3):368-379

127. Wang Y et al (2017) The release and trans-synaptic transmission of Tau via exosomes. Mol Neurodegener 12(1):5

128. Lachenal $\mathrm{G}$ et al (2011) Release of exosomes from differentiated neurons and its regulation by synaptic glutamatergic activity. Mol Cell Neurosci 46(2):409-418

129. Meldolesi J (2018) Exosomes and ectosomes in intercellular communication. Curr Biol 28(8):R435-R444

130. Henderson MX et al (2019) Spread of alpha-synuclein pathology through the brain connectome is modulated by selective vulnerability and predicted by network analysis. Nat Neurosci 22(8):1248-1257
131. Ahmed Z et al (2014) A novel in vivo model of tau propagation with rapid and progressive neurofibrillary tangle pathology: the pattern of spread is determined by connectivity, not proximity. Acta Neuropathol 127(5):667-683

132. Liu L et al (2012) Trans-synaptic spread of tau pathology in vivo. PLoS One 7(2):e31302

133. Guo JL et al (2016) Unique pathological tau conformers from Alzheimer's brains transmit tau pathology in nontransgenic mice. J Exp Med 213(12):2635-2654

134. Boluda S, Iba M, Zhang B, Raible KM, Lee VM, Trojanowski JQ (2015) Differential induction and spread of tau pathology in young PS19 tau transgenic mice following intracerebral injections of pathological tau from Alzheimer's disease or corticobasal degeneration brains. Acta Neuropathol 129(2):221-237

135. Kaufman SK, Thomas TL, Del Tredici K, Braak H, Diamond MI (2017) Characterization of tau prion seeding activity and strains from formaldehyde-fixed tissue. Acta Neuropathol Commun 5(1):41

136. Abounit S, Wu JW, Duff K, Victoria GS, Zurzolo C (2016) Tunneling nanotubes: a possible highway in the spreading of tau and other prion-like proteins in neurodegenerative diseases. Prion 10(5):344-351

137. Rustom A, Saffrich R, Markovic I, Walther P, Gerdes HH (2004) Nanotubular highways for intercellular organelle transport. Science 303(5660):1007-1010

138. Victoria GS, Zurzolo C (2017) The spread of prion-like proteins by lysosomes and tunneling nanotubes: implications for neurodegenerative diseases. J Cell Biol 216(9):2633-2644

139. Rostami J et al (2017) Human astrocytes transfer aggregated alpha-synuclein via tunneling nanotubes. J Neurosci 37(49):11835-11853

140. Tardivel $\mathrm{M}$ et al (2016) Tunneling nanotube (TNT)-mediated neuron-to neuron transfer of pathological Tau protein assemblies. Acta Neuropathol Commun 4(1):117

141. Farhan H (2015) Systems biology of the secretory pathway: what have we learned so far? Biol Cell 107(7):205-217

142. Rabouille C (2017) Pathways of unconventional protein secretion. Trends Cell Biol 27(3):230-240

143. Baker S, Polanco JC, Gotz J (2016) Extracellular vesicles containing P301L mutant tau accelerate pathological tau phosphorylation and oligomer formation but do not seed mature neurofibrillary tangles in ALZ17 mice. J Alzheimers Dis 54(3):1207-1217

144. Polanco JC, Scicluna BJ, Hill AF, Gotz J (2016) Extracellular vesicles isolated from the brains of $\mathrm{rTg} 4510$ mice seed tau protein aggregation in a threshold-dependent manner. J Biol Chem 291(24):12445-12466

145. Saman $\mathrm{S}$ et al (2012) Exosome-associated tau is secreted in tauopathy models and is selectively phosphorylated in cerebrospinal fluid in early Alzheimer disease. J Biol Chem 287(6):3842-3849

146. Winston CN et al (2016) Prediction of conversion from mild cognitive impairment to dementia with neuronally derived blood exosome protein profile. Alzheimers Dement (Amst) 3:63-72

147. Asai $\mathrm{H}$ et al (2015) Depletion of microglia and inhibition of exosome synthesis halt tau propagation. Nat Neurosci 18(11):1584-1593

148. Lee J, Ye Y (2018) The roles of endo-lysosomes in unconventional protein secretion. Cells 7(11):198

149. Fontaine $\mathrm{SN}$ et al (2016) DnaJ/Hsc70 chaperone complexes control the extracellular release of neurodegenerative-associated proteins. EMBO J 35(14): 1537-1549

150. Xu Y et al (2018) DNAJC5 facilitates USP19-dependent unconventional secretion of misfolded cytosolic proteins. Cell Discov $4: 11$ 
151. Lonati E et al (2018) Ischemic conditions affect rerouting of tau protein levels: evidences for alteration in tau processing and secretion in hippocampal neurons. J Mol Neurosci 66(4):604-616

152. Tang $\mathrm{Z}$ et al (2015) mTor mediates tau localization and secretion: implication for Alzheimer's disease. Biochim Biophys Acta 1853(7):1646-1657

153. Rodriguez L, Mohamed NV, Desjardins A, Lippe R, Fon EA, Leclerc N (2017) Rab7A regulates tau secretion. J Neurochem 141(4):592-605

154. Kang S, Son SM, Baik SH, Yang J, Mook-Jung I (2019) Autophagy-mediated secretory pathway is responsible for both normal and pathological tau in neurons. J Alzheimers Dis 70(3):667-680

155. Dujardin S et al (2014) Ectosomes: a new mechanism for nonexosomal secretion of tau protein. PLoS One 9(6):e100760

156. Spitzer $P$ et al (2019) Microvesicles from cerebrospinal fluid of patients with Alzheimer's disease display reduced concentrations of tau and APP protein. Sci Rep 9(1):7089

157. Yan $X$ et al (2016) FRMD4A-cytohesin signaling modulates the cellular release of tau. J Cell Sci 129(10):2003-2015

158. Chai X, Dage JL, Citron M (2012) Constitutive secretion of tau protein by an unconventional mechanism. Neurobiol Dis 48(3):356-366

159. Merezhko $M$ et al (2018) Secretion of tau via an unconventional non-vesicular mechanism. Cell Rep 25(8):2027-2035e4

160. Steringer JP, Nickel W (2018) A direct gateway into the extracellular space: unconventional secretion of FGF2 through selfsustained plasma membrane pores. Semin Cell Dev Biol 83:3-7

161. Mari SA et al (2018) Reversible cation-selective attachment and self-assembly of human tau on supported brain lipid membranes. Nano Lett 18(5):3271-3281

162. Pooler AM, Usardi A, Evans CJ, Philpott KL, Noble W, Hanger DP (2012) Dynamic association of tau with neuronal membranes is regulated by phosphorylation. Neurobiol Aging 33(2):431e27-431e38

163. Ebert AD et al (2010) Tec-kinase-mediated phosphorylation of fibroblast growth factor 2 is essential for unconventional secretion. Traffic 11(6):813-826

164. La Venuta $\mathrm{G}$ et al (2016) Small molecule inhibitors targeting tec kinase block unconventional secretion of fibroblast growth factor 2. J Biol Chem 291(34):17787-17803

165. Kim D et al (2015) Identification of disulfide cross-linked tau dimer responsible for tau propagation. Sci Rep 5:15231

166. Muller HM et al (2015) Formation of disulfide bridges drives oligomerization, membrane pore formation, and translocation of fibroblast growth factor 2 to cell surfaces. J Biol Chem 290(14):8925-8937

167. Gray EG, Paula-Barbosa M, Roher A (1987) Alzheimer's disease: paired helical filaments and cytomembranes. Neuropathol Appl Neurobiol 13(2):91-110

168. Maas T, Eidenmuller J, Brandt R (2000) Interaction of tau with the neural membrane cortex is regulated by phosphorylation at sites that are modified in paired helical filaments. J Biol Chem 275(21):15733-15740

169. Farah CA et al (2006) Tau interacts with Golgi membranes and mediates their association with microtubules. Cell Motil Cytoskelet 63(11):710-724

170. Croft CL et al (2017) Membrane association and release of wildtype and pathological tau from organotypic brain slice cultures. Cell Death Dis 8(3):e2671

171. Barre P, Eliezer D (2006) Folding of the repeat domain of tau upon binding to lipid surfaces. J Mol Biol 362(2):312-326

172. Elbaum-Garfinkle S, Ramlall T, Rhoades E (2010) The role of the lipid bilayer in tau aggregation. Biophys J 98(11):2722-2730
173. Jones EM et al (2012) Interaction of tau protein with model lipid membranes induces tau structural compaction and membrane disruption. Biochemistry 51(12):2539-2550

174. Dicke SS, Tatge L, Engen PE, Culp M, Masterson LR (2017) Isothermal titration calorimetry and vesicle leakage assays highlight the differential behaviors of tau repeat segments upon interaction with anionic lipid membranes. Biochem Biophys Res Commun 493(4):1504-1509

175. Ait-Bouziad N et al (2017) Discovery and characterization of stable and toxic Tau/phospholipid oligomeric complexes. Nat Commun 8(1): 1678

176. Kawarabayashi T et al (2004) Dimeric amyloid beta protein rapidly accumulates in lipid rafts followed by apolipoprotein E and phosphorylated tau accumulation in the Tg2576 mouse model of Alzheimer's disease. J Neurosci 24(15):3801-3809

177. Chirita CN, Necula M, Kuret J (2003) Anionic micelles and vesicles induce tau fibrillization in vitro. J Biol Chem 278(28):25644-25650

178. Barre P, Eliezer D (2013) Structural transitions in tau k18 on micelle binding suggest a hierarchy in the efficacy of individual microtubule-binding repeats in filament nucleation. Protein Sci 22(8): 1037-1048

179. Kadavath $\mathrm{H}$ et al (2015) Tau stabilizes microtubules by binding at the interface between tubulin heterodimers. Proc Natl Acad Sci USA 112(24):7501-7506

180. Gauthier-Kemper A et al (2018) Annexins A2 and A6 interact with the extreme $\mathrm{N}$ terminus of tau and thereby contribute to tau's axonal localization. J Biol Chem 293(21):8065-8076

181. Lasagna-Reeves CA et al (2014) The formation of tau pore-like structures is prevalent and cell specific: possible implications for the disease phenotypes. Acta Neuropathol Commun 2:56

182. Patel N, Ramachandran S, Azimov R, Kagan BL, Lal R (2015) Ion channel formation by tau protein: implications for Alzheimer's disease and tauopathies. Biochemistry 54(50):7320-7325

183. Ding TT, Lee SJ, Rochet JC, Lansbury PT Jr (2002) Annular alpha-synuclein protofibrils are produced when spherical protofibrils are incubated in solution or bound to brain-derived membranes. Biochemistry 41(32):10209-10217

184. Lasagna-Reeves CA, Glabe CG, Kayed R (2011) Amyloid-beta annular protofibrils evade fibrillar fate in Alzheimer disease brain. J Biol Chem 286(25):22122-22130

185. Goedert M, Jakes R, Spillantini MG, Hasegawa M, Smith MJ, Crowther RA (1996) Assembly of microtubule-associated protein tau into Alzheimer-like filaments induced by sulphated glycosaminoglycans. Nature 383(6600):550-553

186. Hasegawa M, Crowther RA, Jakes R, Goedert M (1997) Alzheimer-like changes in microtubule-associated protein Tau induced by sulfated glycosaminoglycans. Inhibition of microtubule binding, stimulation of phosphorylation, and filament assembly depend on the degree of sulfation. J Biol Chem 272(52):33118-33124

187. Wu JW et al (2013) Small misfolded Tau species are internalized via bulk endocytosis and anterogradely and retrogradely transported in neurons. J Biol Chem 288(3):1856-1870

188. Frost B, Jacks RL, Diamond MI (2009) Propagation of tau misfolding from the outside to the inside of a cell. J Biol Chem 284(19):12845-12852

189. Holmes BB et al (2013) Heparan sulfate proteoglycans mediate internalization and propagation of specific proteopathic seeds. Proc Natl Acad Sci USA 110(33):E3138-E3147

190. Brunello CA, Yan X, Huttunen HJ (2016) Internalized Tau sensitizes cells to stress by promoting formation and stability of stress granules. Sci Rep 6:30498

191. Zeineddine $\mathrm{R}$ et al (2015) SOD1 protein aggregates stimulate macropinocytosis in neurons to facilitate their propagation. Mol Neurodegener 10:57 
192. Hooper NM (2011) Glypican-1 facilitates prion conversion in lipid rafts. J Neurochem 116(5):721-725

193. Rauch JN et al (2018) Tau internalization is regulated by 6- $O$ sulfation on heparan sulfate proteoglycans (HSPGs). Sci Rep $8(1): 6382$

194. Mirbaha H, Holmes BB, Sanders DW, Bieschke J, Diamond MI (2015) Tau trimers are the minimal propagation unit spontaneously internalized to seed intracellular aggregation. J Biol Chem 290(24):14893-14903

195. Evans LD et al (2018) extracellular monomeric and aggregated tau efficiently enter human neurons through overlapping but distinct pathways. Cell Rep 22(13):3612-3624

196. Christianson HC, Svensson KJ, van Kuppevelt TH, Li JP, Belting M (2013) Cancer cell exosomes depend on cell-surface heparan sulfate proteoglycans for their internalization and functional activity. Proc Natl Acad Sci USA 110(43):17380-17385

197. Tian T, Zhu YL, Hu FH, Wang YY, Huang NP, Xiao ZD (2013) Dynamics of exosome internalization and trafficking. J Cell Physiol 228(7):1487-1495

198. Polanco JC, Li C, Durisic N, Sullivan R, Gotz J (2018) Exosomes taken up by neurons hijack the endosomal pathway to spread to interconnected neurons. Acta Neuropathol Commun 6(1):10

199. Gomez-Ramos A, Diaz-Hernandez M, Rubio A, Diaz-Hernandez JI, Miras-Portugal MT, Avila J (2009) Characteristics and consequences of muscarinic receptor activation by tau protein. Eur Neuropsychopharmacol 19(10):708-717

200. Flavin WP et al (2017) Endocytic vesicle rupture is a conserved mechanism of cellular invasion by amyloid proteins. Acta Neuropathol 134(4):629-653

201. Calafate S, Flavin W, Verstreken P, Moechars D (2016) Loss of Bin1 promotes the propagation of tau pathology. Cell Rep 17(4):931-940

202. Varkouhi AK, Scholte M, Storm G, Haisma HJ (2011) Endosomal escape pathways for delivery of biologicals. J Control Release 151(3):220-228

203. Margittai M, Langen R (2004) Template-assisted filament growth by parallel stacking of tau. Proc Natl Acad Sci USA 101(28):10278-10283

204. Congdon EE, Kim S, Bonchak J, Songrug T, Matzavinos A, Kuret J (2008) Nucleation-dependent tau filament formation: the importance of dimerization and an estimation of elementary rate constants. J Biol Chem 283(20):13806-13816

205. Fu Z, Aucoin D, Davis J, Van Nostrand WE, Smith SO (2015) Mechanism of nucleated conformational conversion of Abeta42. Biochemistry 54(27):4197-4207

206. Serio TR et al (2000) Nucleated conformational conversion and the replication of conformational information by a prion determinant. Science 289(5483):1317-1321

207. Lasagna-Reeves CA, Castillo-Carranza DL, Guerrero-Muoz MJ, Jackson GR, Kayed R (2010) Preparation and characterization of neurotoxic tau oligomers. Biochemistry 49(47):10039-10041

208. Molliex A et al (2015) Phase separation by low complexity domains promotes stress granule assembly and drives pathological fibrillization. Cell 163(1):123-133

209. Patel A et al (2015) A liquid-to-solid phase transition of the ALS protein FUS accelerated by disease mutation. Cell 162(5):1066-1077

210. Li HR, Chen TC, Hsiao CL, Shi L, Chou CY, Huang JR (2017) The physical forces mediating self-association and phase-separation in the C-terminal domain of TDP-43. Biochim Biophys Acta 1886(2):214-223

211. Ambadipudi S, Biernat J, Riedel D, Mandelkow E, Zweckstetter M (2017) Liquid-liquid phase separation of the microtubulebinding repeats of the Alzheimer-related protein Tau. Nat Commun 8(1):275
212. Boyko S, Qi X, Chen TH, Surewicz K, Surewicz WK (2019) Liquid-liquid phase separation of tau protein: the crucial role of electrostatic interactions. J Biol Chem 294(29):11054-11059

213. Maziuk B, Ballance HI, Wolozin B (2017) Dysregulation of RNA binding protein aggregation in neurodegenerative disorders. Front Mol Neurosci 10:89

214. Anderson P, Kedersha N (2008) Stress granules: the Tao of RNA triage. Trends Biochem Sci 33(3):141-150

215. Vanderweyde $T$ et al (2016) Interaction of tau with the RNAbinding protein TIA1 regulates tau pathophysiology and toxicity. Cell Rep 15(7):1455-1466

216. Apicco DJ et al (2018) Reducing the RNA binding protein TIA1 protects against tau-mediated neurodegeneration in vivo. Nat Neurosci 21(1):72-80

217. Colonna M, Butovsky O (2017) Microglia function in the central nervous system during health and neurodegeneration. Annu Rev Immunol 35:441-468

218. Ferrer I et al (2014) Glial and neuronal tau pathology in tauopathies: characterization of disease-specific phenotypes and tau pathology progression. J Neuropathol Exp Neurol 73(1):81-97

219. Hopp SC et al (2018) The role of microglia in processing and spreading of bioactive tau seeds in Alzheimer's disease. J Neuroinflamm 15(1):269

220. Luo W, Liu W, Hu X, Hanna M, Caravaca A, Paul SM (2015) Microglial internalization and degradation of pathological tau is enhanced by an anti-tau monoclonal antibody. Sci Rep 5:11161

221. Bolos M, Llorens-Martin M, Jurado-Arjona J, Hernandez F, Rabano A, Avila J (2016) Direct evidence of internalization of tau by microglia in vitro and in vivo. J Alzheimers Dis 50(1):77-87

222. Romero-Molina C et al (2018) Distinct microglial responses in two transgenic murine models of tau pathology. Front Cell Neurosci 12:421

223. Maphis $\mathrm{N}$ et al (2015) Reactive microglia drive tau pathology and contribute to the spreading of pathological tau in the brain. Brain 138(Pt 6):1738-1755

224. Perea JR, Lopez E, Diez-Ballesteros JC, Avila J, Hernandez F, Bolos M (2019) Extracellular monomeric tau is internalized by astrocytes. Front Neurosci 13:442

225. Martini-Stoica $\mathrm{H}$ et al (2018) TFEB enhances astroglial uptake of extracellular tau species and reduces tau spreading. J Exp Med 215(9):2355-2377

226. Cruchaga C et al (2013) GWAS of cerebrospinal fluid tau levels identifies risk variants for Alzheimer's disease. Neuron 78(2):256-268

227. Cruchaga C et al (2010) SNPs associated with cerebrospinal fluid phospho-tau levels influence rate of decline in Alzheimer's disease. PLoS Genet 6(9):e1001101

228. Sepulveda-Diaz JE et al (2015) HS3ST2 expression is critical for the abnormal phosphorylation of tau in Alzheimer's diseaserelated tau pathology. Brain 138(Pt 5):1339-1354

229. Huynh MB et al (2019) Glycosaminoglycans from Alzheimer's disease hippocampus have altered capacities to bind and regulate growth factors activities and to bind tau. PLoS One 14(1):e0209573

230. Iliff JJ et al (2014) Impairment of glymphatic pathway function promotes tau pathology after traumatic brain injury. J Neurosci 34(49):16180-16193

231. Iliff JJ et al (2012) A paravascular pathway facilitates CSF flow through the brain parenchyma and the clearance of interstitial solutes, including amyloid beta. Sci Transl Med 4(147):147ra111

232. Patel TK et al (2019) Dural lymphatics regulate clearance of extracellular tau from the CNS. Mol Neurodegener 14(1):11

233. Rasmussen MK, Mestre H, Nedergaard M (2018) The glymphatic pathway in neurological disorders. Lancet Neurol 17(11):1016-1024 
234. Jiang Q et al (2017) Impairment of the glymphatic system after diabetes. J Cereb Blood Flow Metab 37(4):1326-1337

235. Holth JK et al (2019) The sleep-wake cycle regulates brain interstitial fluid tau in mice and CSF tau in humans. Science 363(6429):880-884

236. Zhu Y et al (2018) Chronic sleep disruption advances the temporal progression of tauopathy in P301S mutant mice. J Neurosci 38(48): 10255-10270

237. Vossel KA, Tartaglia MC, Nygaard HB, Zeman AZ, Miller BL (2017) Epileptic activity in Alzheimer's disease: causes and clinical relevance. Lancet Neurol 16(4):311-322

238. Verret L et al (2012) Inhibitory interneuron deficit links altered network activity and cognitive dysfunction in Alzheimer model. Cell 149(3):708-721

239. Masters CL, Bateman R, Blennow K, Rowe CC, Sperling RA, Cummings JL (2015) Alzheimer's disease. Nat Rev Dis Primers 1:15056

240. Williams DR, Lees AJ (2009) Progressive supranuclear palsy: clinicopathological concepts and diagnostic challenges. Lancet Neurol 8(3):270-279

241. Kouri N et al (2011) Neuropathological features of corticobasal degeneration presenting as corticobasal syndrome or Richardson syndrome. Brain 134(Pt 11):3264-3275

242. Dickson DW (2001) Neuropathology of Pick's disease. Neurology 56(11 Suppl 4):S16-S20

243. Steele JC (2005) Parkinsonism-dementia complex of Guam. Mov Disord 20(Suppl 12):S99-S107

244. Karch CM, Jeng AT, Goate AM (2012) Extracellular tau levels are influenced by variability in Tau that is associated with tauopathies. J Biol Chem 287(51):42751-42762

245. Wadhwani AR, Affaneh A, Van Gulden S, Kessler JA (2019) Neuronal apolipoprotein E4 increases cell death and phosphorylated tau release in alzheimer disease. Ann Neurol 85(5):726-739

246. Simon D, Garcia-Garcia E, Royo F, Falcon-Perez JM, Avila J (2012) Proteostasis of tau. Tau overexpression results in its secretion via membrane vesicles. FEBS Lett 586(1):47-54

247. Bilousova $\mathrm{T}$ et al (2018) Suppression of tau propagation using an inhibitor that targets the DK-switch of nSMase2. Biochem Biophys Res Commun 499(4):751-757

248. Winston $\mathrm{CN}$ et al (2019) Neuronal exosome-derived human tau is toxic to recipient mouse neurons in vivo. J Alzheimers Dis 67(2):541-553
249. Reilly P et al (2017) Novel human neuronal tau model exhibiting neurofibrillary tangles and transcellular propagation. Neurobiol Dis 106:222-234

250. Guix FX et al (2018) Detection of aggregation-competent tau in neuron-derived extracellular vesicles. Int J Mol Sci 19(3):663

251. Wang B, Han S (2018) Exosome-associated tau exacerbates brain functional impairments induced by traumatic brain injury in mice. Mol Cell Neurosci 88:158-166

252. Fiandaca MS et al (2015) Identification of preclinical Alzheimer's disease by a profile of pathogenic proteins in neurally derived blood exosomes: a case-control study. Alzheimers Dement 11(6):600-607e1

253. Abner EL, Jicha GA, Shaw LM, Trojanowski JQ, Goetzl EJ (2016) Plasma neuronal exosomal levels of Alzheimer's disease biomarkers in normal aging. Ann Clin Transl Neurol 3(5):399-403

254. Goetzl EJ et al (2016) Decreased synaptic proteins in neuronal exosomes of frontotemporal dementia and Alzheimer's disease. FASEB J 30(12):4141-4148

255. Hamlett ED et al (2017) Neuronal exosomes reveal Alzheimer's disease biomarkers in Down syndrome. Alzheimers Dement 13(5):541-549

256. Lee S, Kim W, Li Z, Hall GF (2012) Accumulation of vesicleassociated human tau in distal dendrites drives degeneration and tau secretion in an in situ cellular tauopathy model. Int J Alzheimers Dis 2012:172837

257. Santa-Maria I, Varghese M, Ksiezak-Reding H, Dzhun A, Wang J, Pasinetti GM (2012) Paired helical filaments from Alzheimer disease brain induce intracellular accumulation of Tau protein in aggresomes. J Biol Chem 287(24):20522-20533

258. Wegmann S, Nicholls S, Takeda S, Fan Z, Hyman BT (2016) Formation, release, and internalization of stable tau oligomers in cells. J Neurochem 139(6):1163-1174

259. Faure $\mathrm{J}$ et al (2006) Exosomes are released by cultured cortical neurones. Mol Cell Neurosci 31(4):642-648

Publisher's Note Springer Nature remains neutral with regard to jurisdictional claims in published maps and institutional affiliations. 